\title{
1 The elasticity of individual protocadherin 15 molecules 2 implicates cadherins as the gating springs for hearing
}

3 Tobias F. Bartsch ${ }^{1,}{ }^{*}$, Felicitas E. Hengel ${ }^{1,{ }^{+}}$, Aaron Oswald $^{1,+}$, Gilman Dionne $^{2}$, Iris V. Chipendo ${ }^{1}$,

4 Simranjit S. Mangat ${ }^{1}$, Muhammad El Shatanofy ${ }^{1}$, Lawrence Shapiro ${ }^{2}$, Ulrich Müller $^{3}$, and

$5 \quad$ A. J. Hudspeth 1

6 Hair cells, the sensory receptors of the inner ear, respond to mechanical forces originating from

7 sounds and accelerations ${ }^{1,2}$. An essential feature of each hair cell is an array of filamentous tip

8 links, consisting of the proteins protocadherin 15 (PCDH15) and cadherin $23(\mathrm{CDH} 23)^{3}$, whose

9 tension is thought to directly gate the cell's transduction channels ${ }^{4,5,6}$. These links are

10 considered far too stiff to represent the gating springs that convert hair-bundle displacement

11 into forces capable of opening the channels ${ }^{7,8}$, and no mechanism has been suggested through

12 which tip-link stiffness could be varied to accommodate hair cells of distinct frequency

13 sensitivity in different receptor organs and animals. As a consequence, the gating spring's

14 identity and mechanism of operation remain central questions in sensory neuroscience. Using

15 a high-precision optical trap, we show that an individual monomer of PCDH15 acts as an

16 entropic spring that is much softer than its enthalpic stiffness alone would suggest ${ }^{7,8}$. This low

17 stiffness implies that the protein is a significant part of the gating spring that controls a hair

18 cell's transduction channels. The tip link's entropic nature then allows for stiffness control

19 through modulation of its tension. We find that a PCDH15 molecule is unstable under tension

20 and exhibits a rich variety of reversible unfolding events that are augmented when the $\mathrm{Ca}^{2+}$

21 concentration is reduced to physiological levels. Tip-link tension and $\mathrm{Ca}^{2+}$ concentration are

22 therefore likely parameters through which nature tunes a gating spring's mechanical

23 properties. 
$25{ }^{1}$ Howard Hughes Medical Institute and Laboratory of Sensory Neuroscience, The Rockefeller

26 University, New York, NY 10065, USA. ${ }^{2}$ Department of Biochemistry and Molecular Biophysics,

27 Zuckerman Mind Brain Behavior Institute, Columbia University, New York, NY 10032, USA. ${ }^{3}$ The

28 Solomon Snyder Department of Neuroscience, Johns Hopkins University, Baltimore, MD

29 21205, USA. *E-mail: tbartsch@rockefeller.edu. ${ }^{+}$These authors contributed equally to this

30 work.

31 Mechanically gated ion channels are ubiquitous. In addition to underlying our senses of hearing,

32 balance, and touch, they are involved in the regulation of processes such as muscle extension,

33 blood pressure, pulmonary inflation, and visceral distension. These channels are opened and

34 closed through the action of gating springs, which are elastic elements that are tensioned by

35 mechanical stimulation and in turn communicate stress to the molecular gates of the respective

36 channels. Gating springs accordingly store mechanical energy and use it to regulate channels'

37 open probabilities. For bacterial mechanoreceptors, which respond to osmotic stress, the cellular

38 membrane itself serves as a gating spring ${ }^{9}$. The ubiquitous Piezo channels of vertebrates extend

39 three membrane-embedded arms that likely act as gating springs by flexing in response to

40 membrane stretching ${ }^{10,11}$. Other mechanosensitive channels, such as NOMPC (TRPN1) in

41 Drosophila, appear to be gated by the tension in elastic ankyrin domains ${ }^{12}$.

42 Gating springs were first posited for hair cells of the vertebrate inner ear, the sensors of

43 the auditory and vestibular systems ${ }^{4}$. Each hair cell is surmounted by a hair bundle-a cluster of

44 erect, actin-filled processes termed stereocilia-that is deflected by mechanical stimulation.

45 However, the identity of the gating springs in these cells has remained controversial. A plausible

46 candidate discovered soon after the gating-spring hypothesis was advanced is the tip link.

47 Extending about $150 \mathrm{~nm}$ between the tip of each stereocilium and the side of its longest

48 neighbor, the tip link is positioned to sense the shear between stereocilia when a hair bundle is

49 deflected (Figure $1 a, b)^{5,13}$. The tip link is a dimer of parallel dimers, comprising two PCDH15

50 molecules joined at their amino termini to a pair of $\mathrm{CDH} 23$ molecules through a "handshake" 
51 whose stability depends upon the presence of bound $\mathrm{Ca}^{2+}$ ions (Figure 1c) ${ }^{14}$. The mechanical

52 properties of hair bundles imply a gating-spring stiffness $\mathrm{s}^{15,16}$ on the order of $1 \mathrm{mN} \cdot \mathrm{m}^{-1}$. However,

53 electron-microscopic images suggest that the tip link is relatively rigid ${ }^{17}$ and crystallographic

54 studies and molecular-dynamics simulations of the relevant cadherins support a stiffness fiftyfold

55 as great as that measured ${ }^{7}$. It has therefore been posited that most of a gating spring's elasticity 56 resides at a tip link's two attachments, rather than within the link itself. To clarify the identity of

57 the hair cell's gating spring, we have therefore examined the elastic properties of a tip-link

58 protein.

\section{Measurement of PCDH15's mechanical characteristics}

60 The mechanics of a protein can be tested by tethering it between two surfaces, applying a force

61 that pulls the surfaces apart, and measuring the protein tether's elongation ${ }^{18}$. To explore directly

62 the stiffness of a tip link, we used a high-precision optical trap to determine the mechanical

63 properties of individual molecules of PCDH15. The extracellular portion of PCDH15 consists of

64 eleven extracellular cadherin domains (EC1-EC11) followed by the membrane-proximal PICA

65 domain. PCDH15 forms a homodimer through interfaces in EC3 and in PICA (Figure 1c) ${ }^{19,20}$,

66 hampering our study of the monomeric protein. We therefore introduced a dimerization-

67 disrupting mutation (V250D) ${ }^{19}$ into EC3 and truncated the protein just before the PICA domain

68 (Figure 1d).

Using a short, site-specific anchor at each of the protein's ends, we placed PCDH15

70 between an immobile, $2 \mu \mathrm{m}$-diameter glass pedestal bead and a mobile $1 \mu \mathrm{m}$ probe bead. Short,

71 stiff anchors were necessary to avoid masking the elastic properties of PCDH15 by those of the

72 anchors (Figure 1f). The probe bead was confined in a weak optical trap whose forward-scattered

73 light we collected to determine the three-dimensional position of the bead with sub-nanometer

74 spatial and $1 \mu$ s temporal resolution ${ }^{21,22}$. A second optical trap, displaced by a few hundred

75 nanometers from the first, served to deliver a force stimulus. By increasing the trap's stiffness,

76 we could apply forces up to and exceeding $60 \mathrm{pN}$ to the tethered protein (Figure 1f). 
Cadherin domains manifest a stereotyped, immunoglobulin-like Greek key folding motif and are separated from one another by conserved linkers that each bind up to three $\mathrm{Ca}^{2+}$ ions in a canonical arrangement (Figure $1 \mathrm{e})^{23} \cdot \mathrm{Ca}^{2+}$ binding is thought to rigidify the linker regions and to stabilize the cadherin domains against force-induced unfolding that elongates the molecule ${ }^{24}$.

81 The $\mathrm{Ca}^{2+}$ concentration of the endolymph, the fluid surrounding tip links, varies between different organs and species from tens to hundreds of micromolar ${ }^{25,26}$. Such concentrations are close to the $\mathrm{Ca}^{2+}$ dissociation constants for the various binding sites ${ }^{7,27}$, raising the intriguing possibility

84 that nature adjusts the $\mathrm{Ca}^{2+}$ concentration to tune the mechanics of tip-link cadherins. We 85 therefore explored the behavior of tensioned $\mathrm{PCDH} 15$ at three $\mathrm{Ca}^{2+}$ concentrations: $3 \mathrm{mM}$, to saturate all binding sites; $20 \mu \mathrm{M}$, a concentration that mimics the concentration of $\mathrm{Ca}^{2+}$ in the endolymph of the mammalian cochlea ${ }^{25}$; and zero.

Because structural changes in proteins are stochastic events that are driven by thermal forces, the rate at which external force is applied can dramatically change the mechanical

90 response. If a protein is pulled too fast, thermal forces do not have sufficient time to cause barrier 91 crossing in the protein's energy landscape before very high external forces are reached.

92 Consequently, the forces at which structural changes occur are artificially elevated at high loading

93 rates $^{28}$. Even in the absence of an acoustic stimulus, tip links experience a constant resting

94 tension $^{29}$ that varies with the frequency sensitivity of a hair cell, from $8 \mathrm{pN}$ at $1 \mathrm{kHz}$ to $50 \mathrm{pN}$ at

$954 \mathrm{kHz}$, with possibly even greater tensions at higher frequencies ${ }^{16}$. In each tip link two copies of

96 PCDH15 act in parallel, and each copy assumes half of the tension of the entire link, 4 pN up to

97 at least $25 \mathrm{pN}$. During normal hearing, a sound stimulus then superimposes an oscillation of only

98 a few piconewtons on this resting tension. Our experiments therefore had to apply slowly

99 changing forces to explore the influence of resting tension on the mechanics of PCDH15.

100 We applied force ramps to the single-molecule tethers at a loading rate of $130 \mathrm{pN} \cdot \mathrm{s}^{-1}$

101 (unless noted otherwise) by linearly increasing the spring constant of the stimulus trap (Extended

102 Data Fig. 1). This rate represented a compromise between slow force application and our desire

103 to collect a statistically relevant number of extension-relaxation cycles for each molecule tested 
104 in a reasonable amount of time. For each cycle, we ramped the force up to $60 \mathrm{pN}$ to cover the

105 entire range of physiologically relevant tensions, then returned it at the same rate to a holding

106 level of 2-4 pN. Depending on the $\mathrm{Ca}^{2+}$ concentration, we adjusted the holding level and duration

107 to allow the protein to refold domains after many but not all cycles. The chosen loading rate

108 likewise led to unfolding events in only a subset of cycles. With these parameter choices we were

109 able to trap the protein in a given conformational state for several extension-relaxation cycles,

110 allowing us to precisely characterize the mechanics of each state.

111 Figure 2a-c shows representative examples of individual force-extension relations for $\mathrm{Ca}^{2+}$

112 concentrations of $3 \mathrm{mM}, 20 \mu \mathrm{M}$, and zero. Each curve features a hockey stick-like shape, as

113 expected for the extension of a biopolymer in a heat bath ${ }^{30}$. As we quantify below, this functional

114 shape indicates that entropic effects dominate PCDH15's elastic response. Abrupt, stepwise

115 extensions or "rips" in the force-extension relations correspond to structural changes of the

116 protein under force. In contrast to typical single-molecule experiments, under our loading

117 conditions PCDH15 never fully unfolded during the extension phase of the stimulus. We therefore

118 frequently observed extensional structural changes even in the relaxation phase of the stimulus.

119 For each $\mathrm{Ca}^{2+}$ concentration, a set of conformational changes leads to a modulated occupation

120 of the force-extension state space, which we visualize by overlapping hundreds of extension-

121 relaxation cycles for one representative molecule apiece (Figure $2 d-f$ ).

\section{Conformational changes}

123 Rips in the force-extension relations at physiological forces suggest that PCDH15 exists in

124 different structural states during hearing. At a saturating $\mathrm{Ca}^{2+}$ concentration, the conformational

125 states accessible to the protein are limited: the state-space heatmap reveals only two major 126 configurations (Figure $2 \mathrm{~d}$ ). The second of these is further divided into two sub-states separated

127 by a difference of only a few nanometers in contour length. State 1 reflects the extensibility of

128 fully folded PCDH15 (Extended Data Note 1). By fitting a polymer model to the force-extension

129 relations for five molecules, we find that State 2 arises from a combination of two classes of 
130 conformational changes leading to mean elongations of $4.0 \pm 0.2 \mathrm{~nm}$ and $15.8 \pm 0.7 \mathrm{~nm}$

131 (means \pm SEMs; respectively distributions of $A_{\cup}$ and $B_{\cup}$ in Figure $2 g$; Table 1 ). The structural origin 132 of these conformational changes is unknown and difficult to determine owing to the large size of 133 the protein. We can, however, rule out the unfolding of entire cadherin domains as the origin of 134 the unfolding events. The length of the folded peptide in each of the eleven cadherin domains 135 ranges from 94 to 123 amino acids, with a mean of 104 residues. At a contour length of $0.39 \mathrm{~nm}$ 136 per residue ${ }^{31}$, the unfolding of each cadherin domain is expected to augment the contour length 137 by about $36 \mathrm{~nm}$ : an elongation of $40.6 \mathrm{~nm}$ less $4.5 \mathrm{~nm}$ to account for the loss of the folded 138 cadherin domain. The observed contour-length changes of the elongations $A_{\cup}$ and $B_{\cup}$ therefore 139 represent protein rearrangements less extreme than the unfolding of a cadherin domain. At a 140 saturating $\mathrm{Ca}^{2+}$ concentration and physiological forces, we never observed length changes in the 141 wild-type protein great enough to account for the unfolding of entire cadherin domains. We next asked whether there are unique structural features in PCDH15 that give rise to 143 the elongations $A_{u}$ and $B_{u}$, or whether several different conformational changes, each with a 144 similar contour-length change, underlay the observed distributions. In most of the extension145 relaxation cycles we did not observe more than one of either class of events (Extended Data 146 Fig. 2). The rare occasions in which several events $A_{u}$ or $B_{u}$ were detected in a single trace were 147 not reproducible across proteins or trials and could thus be explained as the rupture of non148 specific interactions between the protein and either of the confining surfaces. We conclude that 149 a single, unique structural alteration of $\mathrm{PCDH} 15$ is responsible for event $\mathrm{A}_{\mathrm{u}}$, whereas a distinct 150 structural change results in event $B_{\cup}$, precluding the occurrence of several events of either type 151 within the same cycle. Interestingly, the force sensitivity of events $A_{\cup}$ is much narrower than that 152 of events $B_{u}$ (Figure $2 \mathrm{j}$ ). From these distributions we determined the statistical dependence of 153 both classes: an event $A_{u}$ generally follows an event $B_{\cup}(p<0.05$ and $p<0.1$ for respectively three 154 and two of the five tested molecules). It is plausible that both structural changes resulted from 155 the same cadherin domain, with elongation $\mathrm{B}_{\mathrm{u}}$ leading to a destabilization that facilitated 156 elongation $\mathrm{Au}$. 
We found the protein in State 1 at the beginning of many extension-relaxation cycles and

158 concluded that there is a high probability of refolding of both event $A_{u}$ and event $B_{u}$ between

159 cycles. We indeed routinely detected refolding events $A_{F}$ during the relaxation phase of our

160 protocol (Figure $2 \mathrm{~g}$ ), but rarely observed refolding events $\mathrm{B}_{\mathrm{F}}$. The latter events probably occurred

161 only at very low forces, for which the slight shortening was lost in Brownian noise (Extended Data

162 Fig. 3).

We next reduced the $\mathrm{Ca}^{2+}$ concentration to the physiological value of $20 \mu \mathrm{M}$ and exposed

164 a tethered PCDH15 protein to the same force protocol. The extension-relaxation cycles showed

165 conformational changes identical to the previously observed classes (Figure $2 \mathrm{~h} ; \mathrm{A}_{u}: 3.9 \pm 0.4 \mathrm{~nm}$,

$166 \mathrm{~B}_{\mathrm{U}}: 15.0 \pm 0.6 \mathrm{~nm}$, means $\left.\pm \mathrm{SEMs}, N=8\right)$. At this $\mathrm{Ca}^{2+}$ concentration, however, an additional class

167 of unfolding events emerged with a contour-length change of $35 \pm 1 \mathrm{~nm}$ (mean $\pm S E M$; $C_{U}$ in

168 Figure $2 \mathrm{~b}, \mathrm{~h})$, in excellent agreement with the elongation expected for unfolding of an entire

169 cadherin domain ${ }^{32}$. At holding forces of 2-4 pN the refolding of cadherin domains was a slow

170 process and occurred on a time scale of seconds, in line with other proteins that feature

171 immunoglobulin-like motifs ${ }^{33}$. In some extension-relaxation cycles we observed the successive

172 unfolding of several cadherin domains (Extended Data Fig. 4). Because unfolding of any of the

173 eleven extracellular cadherin domains should increase the contour length by a similar amount,

174 we could neither assign unfolding events to particular domains nor elucidate the sequence in

175 which the domains unraveled.

The heatmap of all force-extension relations originated from a mixture of the unfolding

177 events $A_{U}, B_{U}$, and $C_{u}$ and their respective refolding events. The contour lengths that gave rise to

178 the annotated States 1 to 8 (Figure 2e) were a consequence of the unfolding of up to three

179 cadherin domains in series with up to one unfolding event of type Bu. Structural changes of type

$180 \mathrm{~A}_{U}$ and $\mathrm{A}_{\mathrm{F}}$, which are clearly visible in the heatmap for a $\mathrm{Ca}^{2+}$ concentration of $3 \mathrm{mM}$ as a 181 subdivision of State 2 (Figure $2 \mathrm{~d}$ ), are not apparent in the state space at $20 \mu \mathrm{M}\left[\mathrm{Ca}^{2+}\right.$ ]. It is 182 possible that there were $\mathrm{Ca}^{2+}$-dependent sub-nanometer changes in the contour length that 183 averaged out small effects of events $A_{U}$ and $A_{F}$ in the heatmap. We again investigated a potential 
184 sequential dependence of unfolding classes by their force distributions (Figure 2k): even though

185 events of type $A_{\cup}$ followed events of type $\mathrm{B}_{\cup}$ at $3 \mathrm{mM}\left[\mathrm{Ca}^{2+}\right]$, at a physiological $\mathrm{Ca}^{2+}$ concentration

186 these structural changes in eight molecules were independent of one another $(p>0.1$ for each

187 molecule). Moreover, events of types $A_{U}$ and $B_{U}$ were also independent of events of type $C_{U}$, the 188 unfolding of entire cadherin domains.

189 We next investigated the mechanics of PCDH15 in the absence of $\mathrm{Ca}^{2+}$. Representative 190 force-extension relations feature a plethora of conformational changes (Figure 2c,i), many of 191 which could no longer be clearly grouped into any of the classes $A_{U, F}$ and $B_{U}$. Events with a mean 192 of $37 \pm 2 \mathrm{~nm}$ (mean \pm SEM for five molecules) continued to characterize a well-defined class Cu.

193 The heatmap of all extension-relaxation cycles had a structure reminiscent of that at a $\mathrm{Ca}^{2+}$ 194 concentration of $20 \mu \mathrm{M}$ (Figure $2 \mathrm{f}$ ). The structure in the absence of $\mathrm{Ca}^{2+}$ arose from the unfolding 195 of a discrete number of cadherin domains in series with the unclassifiable shorter structural 196 changes that likely represented the partial unfolding of one or more domains. This lack of well197 defined short structural changes was also evident from the force distribution of the observed rips 198 (Figure 2l).

\section{Stiffness of PCDH15}

200 It is unknown whether tip-link cadherins are completely or only partially folded during normal 201 hearing. We therefore investigated the stiffness not only of folded PCDH15 but also that of 202 conformational states with a progressively greater number of unfolded domains. The total 203 stiffness of PCDH15 comprises both enthalpic and entropic components, whose contributions we 204 quantified by fitting the force-extension relations with a model of the protein as a freely jointed 205 chain $^{34}$ formed by the eleven folded cadherin domains in series with a worm-like chain ${ }^{35}$ 206 representing the ten unstructured linker regions. We allowed enthalpic extensibility through a 207 Hookean spring constant and included an additional worm-like chain to model any unfolded 208 portions of the protein (Extended Data Fig. 5). Because the unfolded polypeptide chains and the 209 linker regions are structurally similar, we modeled them both with the same persistence length; 
210 fits to the data for thirteen molecules yielded $I p_{\text {peptide }}=0.49 \pm 0.04 \mathrm{~nm}$ (mean $\pm \mathrm{SEM}$ ). For folded

$211 \mathrm{PCDH} 15$ at a $\mathrm{Ca}^{2+}$ concentration of $3 \mathrm{mM}$, we found a length of $2.9 \pm 0.5 \mathrm{~nm}$ for each of the eleven

212 solid segments of the chain, a length of $1.4 \pm 0.5 \mathrm{~nm}$ for each of the ten flexible linkers between

213 the solid segments, and an enthalpic spring constant of $9 \pm 4 \mathrm{mN} \cdot \mathrm{m}^{-1}$ for the Hookean stiffness of

214 the protein (means \pm SEMs, $N=5$ molecules, Table 1). The full length of a solid segment

215 combined with its associated linker region was $4.3 \pm 0.7 \mathrm{~nm}$, in excellent agreement with the

216 value of $4.5 \mathrm{~nm}$ per cadherin repeat from crystal structures of cadherin domains ${ }^{23}$. Much to our

217 surprise, these values did not change when the $\mathrm{Ca}^{2+}$ concentration was lowered to $20 \mu \mathrm{M}$, the

218 physiological level in the cochlea: an elevated $\mathrm{Ca}^{2+}$ concentration stabilizes cadherin domains

219 against unfolding but does not augment the stiffness of the folded protein. The stiffness

220 predicted by our model is in good agreement with the slopes of the different states in the state-

221 space heatmap (Figure 3a,b).

222 Across all states and $\mathrm{Ca}^{2+}$ concentrations the measured and predicted stiffness of the 223 protein is much smaller than its enthalpic stiffness of about $10 \mathrm{mN} \cdot \mathrm{m}^{-1}$. The additional 224 compliance is entropic, arising from the thermal motion of the individual cadherin domains and 225 from thermal undulations in the inter-domain linker regions and unfolded polypeptide chains.

226 When PCDH15 is tensed, this thermal kinking is smoothed out and the protein elongates. The 227 progressive unfolding of domains further softens the protein by introducing additional 228 disordered polypeptide chains (Figure 3b). At high forces most thermal bends have been 229 straightened and the enthalpic elasticity begins to dominate the protein's response. Importantly, 230 we find that for physiological tension the protein's response to force is dominated by entropic 231 elasticity. The protein's stiffness approaches its enthalpic value only for unphysiologically high 232 tensions (Figure 3, Extended Data Fig. 6).

\section{Unfolding of cadherin domains under forces relevant for hearing}

234 Elevated tension not only increases the stiffness of PCDH15 but also heightens the likelihood that 235 entire cadherin domains unfold. Do cadherin domains unfold during normal hearing? If so, do 
236 they refold under physiological conditions, or could tip links with persistently unstructured

237 regions exist in vivo?

238 To determine the unfolding rate of cadherin domains under physiological tensions, we

239 transformed the force distributions of type $C_{u}$ domain-unfolding events into unfolding rates as a

240 function of constant force (Figure 4) ${ }^{28}$. For a given unfolding event we could not determine which

241 of the eleven cadherin domains had unfolded, so our result was an average over several or all of

242 the domains. The transformation additionally assumed that there was no cooperativity between

243 the unfolding of individual domains. If the unfolding of one domain were to increase the

244 probability that an adjacent domain would unravel, for example, our computed unfolding rates

245 would have systematically overestimated the rate at which fully folded tip links unfold.

246 We computed unfolding rates for $\mathrm{Ca}^{2+}$ concentrations of both $20 \mu \mathrm{M}$ and zero (Figure 4).

247 As expected, the presence of $\mathrm{Ca}^{2+}$ stabilized cadherin against force-induced unfolding: in the ion's

248 absence the domains unfolded many times faster than in its presence. With a physiological $\mathrm{Ca}^{2+}$

249 concentration and at $20 \mathrm{pN}$ of tension, the upper range of physiological resting values, a single

250 cadherin domain unfolded at a rate of approximately once every $100 \mathrm{~s}$. A fully folded PCDH

251 molecule-consisting of 11 domains-then unfolded a domain every $10 \mathrm{~s}$. At even higher

252 tensions this rate rapidly increased, to roughly $0.4 \mathrm{~s}^{-1}$ for the unfolding of a single cadherin

253 domain at a tension of $60 \mathrm{pN}$. Because unfolding events at forces of $10-20 \mathrm{pN}$ were extremely

254 rare and might have corresponded to transitions from molten-globule states (Extended Data

255 Note 2), we were unable to reliably calculate force-dependent unfolding rates for this force

256 range. Extrapolation of the available data nevertheless suggests rates of approximately $0.003 \mathrm{~s}^{-1}$

257 at $10 \mathrm{pN}$ of tension. These results indicate that unfolding of cadherin domains would not take

258 place within individual cycles of an auditory stimulus: for physiological forces the unfolding rate

259 is much too low to follow stimuli with frequencies ranging from hundreds to thousands of hertz.

260 However, provided that at the link's resting tension a domain's unfolding rate exceeds its

261 refolding rate, cadherin domains in a tensed tip link could exist in a permanently unfolded state.

262 We never observed refolding of cadherin domains during any of the recorded extension- 
263 relaxation cycles, even though such events should be easily detectable at tensions exceeding

2644 pN (Extended Data Fig. 3). Refolding instead occurred only during the holding phase between

265 successive cycles, provided the holding force was below $4 \mathrm{pN}$ and the waiting time was on the

266 order of several seconds. We conclude that for tensions higher than $20 \mathrm{pN}$ per molecule the

267 unfolding rate-although very small-exceeds the refolding rate. Our data indicate that this is

268 also the case for the force range of 4-20 pN, but owing to the possible influence of molten-globule

269 states we could not determine this with certainty. Our result suggests that some cadherin

270 domains in tip links in vivo exist in a perpetually unfolded state. Such unfolded states would

271 decrease the protein's stiffness (Figure $3 b$ ) and could be a mechanism by which a tip link softens

272 even under high tension.

273 The critical force at which the unfolding and refolding rates of a cadherin domain are

274 equal remains to be determined. However, the giant muscle protein titin, which has

275 immunoglobin folds similar to those of cadherin, exhibits a critical force ${ }^{33}$ of $5.4 \mathrm{pN}$. If cadherin

276 domains feature a similar value, the resting tensions in low-frequency hair cells might be less

277 than the critical force and bias the tip link's domains towards a fully folded state, whereas the tip

278 link might occur in a partially unfolded state in hair cells sensitive to high frequencies.

Effect of a mutation associated with hearing loss

280 Over one hundred mutations of the tip-link cadherins cause hearing loss in humans ${ }^{36}$. The 281 deletion of residue V767 in EC7 is particularly interesting, for it leads to deafness -stemming

282 from a deficit in the cochlea, with its low $\mathrm{Ca}^{2+}$ concentration-but not a loss of function in the 283 vestibular labyrinth-which enjoys a higher $\mathrm{Ca}^{2+}$ concentration ${ }^{37}$. This mutation evidently does 284 not hinder tip-link formation, but might change the elastic properties of the link.

285 We investigated how this deletion affects the mechanical properties of monomeric PCDH15 (V250D, $\triangle$ V767) and found that there was a small but detectable probability that force 287 unfolded a complete cadherin domain even when PCDH15 was saturated with $\mathrm{Ca}^{2+}$ (Figure 5). 288 During identical treatment of $\mathrm{Ca}^{2+}$-saturated proteins without pathologic mutations we never 
289 observed the unfolding of complete domains (Figure $2 \mathrm{~g}$ ). By shortening one strand of EC7, the

290 mutation likely caused a slight misalignment of amino-acid residues and thus destabilized the

291 domain. When we performed experiments at the physiological $\mathrm{Ca}^{2+}$ concentration of $20 \mu \mathrm{M}$, we

292 could not detect a difference in domain unfolding between the mutant and wild-type proteins.

\section{Discussion}

294 The behavior of a gated ion channel is usually binary: the channel is open or closed. A 295 mechanically activated channel can nevertheless signal fine nuances of a stimulus by rapidly

296 fluttering between the two states, such that the average open probability provides a smoothly 297 graded representation of the stimulus. A gating spring makes this possible: tensed by a stimulus 298 and battered by thermal noise, the spring continuously adjusts the open probability of the 299 associated channel over a significant range of inputs. This range is determined by the gating 300 spring's stiffness, and thus by such molecular details as the entropic elasticity and folding 301 transitions demonstrated here.

302 The stiffness of gating springs in outer hair cells increases with heightened resting tension 303 along the tonotopic axis ${ }^{16}$, from $1.9 \mathrm{mN} \cdot \mathrm{m}^{-1}$ at $7 \mathrm{pN}$ of tension to $5.5 \mathrm{mN} \cdot \mathrm{m}^{-1}$ for $50 \mathrm{pN}$. 304 Simulations of short segments of tip-link proteins indicated that they are orders of magnitudes 305 too stiff to account for these values ${ }^{7,8}$. However, our single-molecule experiments on the 306 extracellular domain of $\mathrm{PCDH} 15$ reveal that the protein has a stiffness comparable to that of 307 gating springs in vivo and displays similar strain-hardening. Across the physiological force range 308 most of PCDH15's compliance is of entropic origin; the protein's enthalpic stiffness of $9 \mathrm{mN} \cdot \mathrm{m}^{-1}$ 309 emerges only at very high tension. Our stiffness values (Figure 3) are systematically lower than 310 those found for gating springs in vivo, which is not surprising because our measurements tested 311 only a monomer of one constituent protein. The dimeric arrangement of PCDH15 roughly 312 doubles the enthalpic stiffness of the monomer. Moreover, a tip link adopts a helical structure $\mathrm{e}^{17,19}$ 313 that likely reduces the magnitude of its thermal undulations, decreasing the entropic 314 contribution to the tip link's mechanics and further increasing its stiffness. Finally, the 
315 arrangement of a dimer of $\mathrm{CDH} 23$ in series with the $\mathrm{PCDH} 15$ dimer is expected to reduce the

316 stiffness by about $70 \%$. At very high tensions, when entropic effects are largely suppressed and

317 enthalpy dominates, we estimate that the stiffness of the full-length, dimeric tip link is $6 \mathrm{mN} \cdot \mathrm{m}^{-1}$

318 (Extended Data Note 3), a value in good agreement with the stiffness of strongly tensioned gating

319 springs in vivo. These results suggest that the tip-link cadherins are a major component of the

320 gating spring for mechanotransduction in hair cells.

321 The stiffness of hair bundles increases at low $\mathrm{Ca}^{2+}$ concentrations ${ }^{38}$, an observation that

322 can now be interpreted as strain-hardening of the tip links. Low $\mathrm{Ca}^{2+}$ levels cause molecular

323 motors to upregulate tip-link tension, which suppresses each link's thermal motion and increases

324 its stiffness. In addition to this indirect modulation of tip-link stiffness by the $\mathrm{Ca}^{2+}$ concentration,

325 we found that $\mathrm{Ca}^{2+}$ also directly affects the rate at which cadherin domains unfold under force.

326 At a tension of $20 \mathrm{pN}$ and at a $\mathrm{Ca}^{2+}$ concentration of $20 \mu \mathrm{M}$, individual cadherin domains unfold

327 an order of magnitude more slowly than in the absence of $\mathrm{Ca}^{2+}$. We never observed the unfolding

328 of entire cadherin domains at a $\mathrm{Ca}^{2+}$ concentration of $3 \mathrm{mM}$. The sensitivity of PCDH15 to $\mathrm{Ca}^{2+}$

329 suggests that the variable concentrations of the ion in different receptor organs tunes the

330 mechanical properties of tip links and thus of hair bundles to the organs' specific requirements.

331 Even within a single organ, the guinea pig's cochlea, the $\mathrm{Ca}^{2+}$ concentration increases fourfold

332 along the tonotopic axis from base to apex ${ }^{26}$. This gradient might adjust tip-link stiffness to accord

333 with the frequency response of the individual hair cells. Finally, hair cells can enhance the local

$334 \mathrm{Ca}^{2+}$ concentration around their hair bundles through the activity of membrane $\mathrm{Ca}^{2+}$ pumps $^{39}$.

335 This phenomenon raises the interesting possibility that the stiffness of tip links is modulated by

336 the locally varying $\mathrm{Ca}^{2+}$ concentration in response to hair-cell activity.

337 We found that the elastic properties of folded PCDH15 are surprisingly independent of

338 the $\mathrm{Ca}^{2+}$ concentration. This result seems to contradict the impression conveyed by electron-

339 microscopic images ${ }^{3}$, in which cadherins transition from a disordered globular conformation to a

340 rod-like chain of domains in the presence of progressively larger amounts of $\mathrm{Ca}^{2+}$. Disordered

341 states should make larger entropic contributions to PCDH15's elasticity than ordered states, a 
342 difference not apparent in our data. Note, however, that the divergence between electron-

343 microscopic images and single-molecule data has also been observed for other proteins such as

$344 \operatorname{titin}^{40}$. A possible explanation is that the configurations of proteins adsorbed to electron-

345 microscopic substrates are far from their equilibrium conformations, so that the variations in

346 shape do not accurately capture the thermal motion in solution ${ }^{41}$.

347 The force-extension relations of hair bundles reveal that, for unphysiologically large

348 stimuli, gating springs can stretch ${ }^{42}$ by at least $120 \mathrm{~nm}$, a value thought to be incompatible with

349 the extensibility of tip-link cadherins. It has consequently been suggested that the gating spring's

350 stiffness stems from the elasticity of the plasma membrane or cytoskeleton into which the tip

351 links insert ${ }^{43}$. We have shown that invoking such sources of elasticity is unnecessary: cadherin

352 domains in the tip-link proteins can unfold under physiological stimuli, albeit at a low rate, and

353 such unfolding events become very likely at high forces. Extension of the tip link by $120 \mathrm{~nm}$ is

354 easily possible through the unfolding of several cadherin domains. In further support of domain

355 unfolding, the length distribution of tip links in the bullfrog's hair bundles, as determined by

356 electron-microscopic tomography, features two distinct classes ${ }^{13}$ with means near $110 \mathrm{~nm}$ and

$357170 \mathrm{~nm}$. The unfolding of two cadherin domains per tip-link monomer could account for this

358 length difference. Such unfolding events could soften the gating spring at high resting tensions

359 and protect both the tip link and the associated mechanotransduction machinery from damage

360 during loud sounds.

361 In addition to the unfolding of entire cadherin domains, we also observed partial domain

362 unfolding with contour length increases of $4 \mathrm{~nm}$ and $15 \mathrm{~nm}$. Future single-molecule work will be

363 necessary to elucidate the structural correlates of these conformational changes and to

364 determine what role they play in hearing. Additional experiments will also be necessary to test

365 the stiffness of PCDH15 dimers and of the full tip link and to confirm that domain unfolding occurs

366 for those constructs and for tip links in vivo. 


\section{References}

1. Hudspeth, A. J. Integrating the active process of hair cells with cochlear function. Nat. Rev. Neurosci. 15, 600-614 (2014).

2. Reichenbach, T. \& Hudspeth, A. J. The physics of hearing: fluid mechanics and the active process of the inner ear. Rep. Prog. Phys. Phys. Soc. G. B. 77, 076601 (2014).

3. Kazmierczak, P. et al. Cadherin 23 and protocadherin 15 interact to form tip-link filaments in sensory hair cells. Nature 449, 87-91 (2007).

4. Corey, D. P. \& Hudspeth, A. J. Kinetics of the receptor current in bullfrog saccular hair cells. J. Neurosci. Off. J. Soc. Neurosci. 3, 962-976 (1983).

5. Pickles, J. O., Comis, S. D. \& Osborne, M. P. Cross-links between stereocilia in the guinea pig organ of Corti, and their possible relation to sensory transduction. Hear. Res. 15, 103-112 (1984).

6. Assad, J. A., Shepherd, G. M. \& Corey, D. P. Tip-link integrity and mechanical transduction in vertebrate hair cells. Neuron 7, 985-994 (1991).

7. Sotomayor, M., Weihofen, W. A., Gaudet, R. \& Corey, D. P. Structural determinants of cadherin-23 function in hearing and deafness. Neuron 66, 85-100 (2010).

8. Sotomayor, M., Corey, D. P. \& Schulten, K. In Search of the Hair-Cell Gating Spring. Structure 13, 669-682 (2005).

9. Wiggins, P. \& Phillips, R. Analytic models for mechanotransduction: Gating a mechanosensitive channel. Proc. Natl. Acad. Sci. 101, 4071-4076 (2004).

10. Saotome, K. et al. Structure of the mechanically activated ion channel Piezo1. Nature 554, 481-486 (2018).

11. Zhao, Q. et al. Structure and mechanogating mechanism of the Piezo1 channel. Nature 554, 487-492 (2018).

12. Jin, P. et al. Electron cryo-microscopy structure of the mechanotransduction channel NOMPC. Nature 547, 118-122 (2017). 
394 13. Auer, M. et al. Three-dimensional architecture of hair-bundle linkages revealed by electron395 microscopic tomography. J. Assoc. Res. Otolaryngol. JARO 9, 215-224 (2008).

14. Sotomayor, M., Weihofen, W. A., Gaudet, R. \& Corey, D. P. Structure of a force-conveying cadherin bond essential for inner-ear mechanotransduction. Nature 492, 128-132 (2012).

15. Howard, J. \& Hudspeth, A. J. Compliance of the hair bundle associated with gating of mechanoelectrical transduction channels in the bullfrog's saccular hair cell. Neuron 1, 189199 (1988).

16. Tobin, M., Michel, V., Michalski, N., Martin, P. Tonotopy of the mammalian cochlea is associated with stiffness and tension gradients of the hair cell's tip-link complex (2018) doi.org/10.1101/497222.

17. Kachar, B., Parakkal, M., Kurc, M., Zhao, Y. \& Gillespie, P. G. High-resolution structure of hair-cell tip links. Proc. Natl. Acad. Sci. U. S. A. 97, 13336-13341 (2000).

18. Smith, S., Finzi, L. \& Bustamante, C. Direct mechanical measurements of the elasticity of single DNA molecules by using magnetic beads. Science 258, 1122-1126 (1992).

19. Dionne, G. et al. Mechanotransduction by PCDH15 Relies on a Novel cis-Dimeric Architecture. Neuron 99, 480-492.e5 (2018).

20. Ge, J. et al. Structure of mouse protocadherin 15 of the stereocilia tip link in complex with LHFPL5. eLife 7, (2018).

21. Pralle, A., Prummer, M., Florin, E.-L., Stelzer, E. H. K. \& H rber, J. K. H. Three-dimensional high-resolution particle tracking for optical tweezers by forward scattered light. Microsc. Res. Tech. 44, 378-386 (1999).

22. Bartsch, T. F., Kochanczyk, M. D., Lissek, E. N., Lange, J. R. \& Florin, E.-L. Nanoscopic imaging of thick heterogeneous soft-matter structures in aqueous solution. Nat. Commun. 7 , (2016).

23. Shapiro, L. et al. Structural basis of cell-cell adhesion by cadherins. Nature 374, 327-337 (1995). 
420

421

422

423

424

425

426

427

428

429

430

431

432

433

434

435

436

437

24. Nagar, B., Overduin, M., Ikura, M. \& Rini, J. M. Structural basis of calcium-induced Ecadherin rigidification and dimerization. Nature 380, 360-364 (1996).

25. Bosher, S. K. \& Warren, R. L. Very low calcium content of cochlear endolymph, an extracellular fluid. Nature 273, 377-378 (1978).

26. Salt, A. N., Inamura, N., Thalmann, R. \& Vora, A. Calcium gradients in inner ear endolymph. Am. J. Otolaryngol. 10, 371-375 (1989).

27. Powers, R. E., Gaudet, R. \& Sotomayor, M. A Partial Calcium-Free Linker Confers Flexibility to Inner-Ear Protocadherin-15. Structure 25, 482-495 (2017).

28. Zhang, Y. \& Dudko, O. K. A transformation for the mechanical fingerprints of complex biomolecular interactions. Proc. Natl. Acad. Sci. U. S. A. 110, 16432-16437 (2013).

29. Jaramillo, F. \& Hudspeth, A. J. Displacement-clamp measurement of the forces exerted by gating springs in the hair bundle. Proc. Natl. Acad. Sci. U. S. A. 90, 1330-1334 (1993).

30. Marko, J. F. \& Siggia, E. D. Fluctuations and supercoiling of DNA. Science 265, 506-508 (1994).

31. Ainavarapu, S. R. K. et al. Contour length and refolding rate of a small protein controlled by engineered disulfide bonds. Biophys. J. 92, 225-233 (2007).

32. Oroz, J. et al. Nanomechanics of the Cadherin Ectodomain: "CANALIZATION" BY Ca ${ }^{2+}$ BINDING RESULTS IN A NEW MECHANICAL ELEMENT. J. Biol. Chem. 286, 94059418 (2011).

33. Chen, H. et al. Dynamics of equilibrium folding and unfolding transitions of titin immunoglobulin domain under constant forces. J. Am. Chem. Soc. 137, 3540-3546 (2015).

34. Rubinstein, M. \& Colby, R. H. Polymer physics. (Oxford University Press, 2003).

35. Marko, J. F. \& Siggia, E. D. Stretching DNA. Macromolecules 28, 8759-8770 (1995).

36. Jaiganesh, A. et al. Zooming in on Cadherin-23: Structural Diversity and Potential Mechanisms of Inherited Deafness. Struct. Lond. Engl. 1993 26, 1210-1225.e4 (2018). 
445

446

447

37. Zhan, Y., Liu, M., Chen, D., Chen, K. \& Jiang, H. Novel mutation located in EC7 domain of protocadherin-15 uncovered by targeted massively parallel sequencing in a family segregating non-syndromic deafness DFNB23. Int. J. Pediatr. Otorhinolaryngol. 79, 983-986 (2015).

38. Marquis, R. E. \& Hudspeth, A. J. Effects of extracellular Ca2+ concentration on hair-bundle stiffness and gating-spring integrity in hair cells. Proc. Natl. Acad. Sci. U. S. A. 94, 1192311928 (1997).

39. Yamoah, E. N. et al. Plasma membrane Ca2+-ATPase extrudes Ca2+ from hair cell stereocilia. J. Neurosci. Off. J. Soc. Neurosci. 18, 610-624 (1998).

40. Leake, M. C., Wilson, D., Gautel, M. \& Simmons, R. M. The Elasticity of Single Titin Molecules Using a Two-Bead Optical Tweezers Assay. Biophys. J. 87, 1112-1135 (2004).

41. Broedersz, C. P. \& MacKintosh, F. C. Modeling semiflexible polymer networks. Rev. Mod. Phys. 86, 995-1036 (2014).

42. Shepherd, G. M. \& Corey, D. P. The extent of adaptation in bullfrog saccular hair cells. $J$. Neurosci. Off. J. Soc. Neurosci. 14, 6217-6229 (1994).

43. Powers, R. J. et al. Stereocilia membrane deformation: implications for the gating spring and mechanotransduction channel. Biophys. J. 102, 201-210 (2012).

44. Bell, G. Models for the specific adhesion of cells to cells. Science 200, 618-627 (1978).

45. Gibson, D. G. Enzymatic Assembly of Overlapping DNA Fragments. in Methods in Enzymology 498, 349-361 (Elsevier, 2011).

46. Aricescu, A. R., Lu, W. \& Jones, E. Y. A time- and cost-efficient system for high-level protein production in mammalian cells. Acta Crystallogr. D Biol. Crystallogr. 62, 1243-1250 (2006).

47. Zakeri, B. et al. Peptide tag forming a rapid covalent bond to a protein, through engineering a bacterial adhesin. Proc. Natl. Acad. Sci. U. S. A. 109, E690-697 (2012).

48. Pincet, F. \& Husson, J. The Solution to the Streptavidin-Biotin Paradox: The Influence of History on the Strength of Single Molecular Bonds. Biophys. J. 89, 4374-4381 (2005).

49. Zhu, M., Lerum, M. Z. \& Chen, W. How To Prepare Reproducible, Homogeneous, and Hydrolytically Stable Aminosilane-Derived Layers on Silica. Langmuir 28, 416-423 (2012). 
472

473

474

475

476

477

478

479

480

481

482

483

50. Jahn, M. et al. The charged linker of the molecular chaperone Hsp90 modulates domain contacts and biological function. Proc. Natl. Acad. Sci. 111, 17881-17886 (2014).

51. Swoboda, M. et al. Enzymatic Oxygen Scavenging for Photostability without pH Drop in Single-Molecule Experiments. ACS Nano 6, 6364-6369 (2012).

52. Bartsch, T. F. et al. Detecting Sequential Bond Formation Using Three-Dimensional Thermal Fluctuation Analysis. ChemPhysChem 10, 1541-1547 (2009).

53. Kochanczyk, M. D., Bartsch, T. F., Taute, K. M. \& Florin, E.-L. Power spectral density integration analysis and its application to large bandwidth, high precision position measurements. in (eds. Dholakia, K. \& Spalding, G. C.) $84580 \mathrm{H}$ (2012). doi:10.1117/12.929349

54. Neuman, K. C., Chadd, E. H., Liou, G. F., Bergman, K. \& Block, S. M. Characterization of Photodamage to Escherichia coli in Optical Traps. Biophys. J. 77, 2856-2863 (1999).

55. Tischer, C., Pralle, A. \& Florin, E.-L. Determination and Correction of Position Detection Nonlinearity in Single Particle Tracking and Three-Dimensional Scanning Probe Microscopy. Microsc. Microanal. 10, 425-434 (2004).

56. Monkos, K. Viscosity of bovine serum albumin aqueous solutions as a function of temperature and concentration. Int. J. Biol. Macromol. 18, 61-68 (1996).

57. Berg-Sørensen, K. \& Flyvbjerg, H. Power spectrum analysis for optical tweezers. Rev. Sci. Instrum. 75, 594-612 (2004).

58. Block, S. M., Goldstein, L. S. B. \& Schnapp, B. J. Bead movement by single kinesin molecules studied with optical tweezers. Nature 348, 348-352 (1990).

59. Petrosyan, R. Improved approximations for some polymer extension models. Rheol. Acta 56, 21-26 (2017).

60. Rivas-Pardo, J. A. et al. Work Done by Titin Protein Folding Assists Muscle Contraction. Cell Rep. 14, 1339-1347 (2016).

61. Elms, P. J., Chodera, J. D., Bustamante, C. \& Marqusee, S. The molten globule state is unusually deformable under mechanical force. Proc. Natl. Acad. Sci. 109, 3796-3801 (2012). 
499 Acknowledgments The authors thank Julio Fernandez and Jaime Andrés Rivas-Pardo for 500 guidance concerning the chemical functionalization of glass substrates; Brian Fabella and Vadim

501 Sherman for high-precision fabrication; Brandon Razooky and Maria Vologodskaia for assistance

502 with cloning; and Anna Kaczynska for help with protein expression. This work was partially 503 supported by a Junior Fellow award from the Simons Foundation and a Pilot Grant from the Kavli 504 Foundation to T.F.B. A.J.H. is an Investigator of Howard Hughes Medical Institute.

505 Author contributions T.F.B. and A.J.H designed the project. T.F.B., F.E.H., A.O., S.S.M., M.E.S., 506 and U.M. created cDNA clones for PCDH15. G.D. and L.S. expressed and purified the recombinant 507 protein. I.V.C., T.F.B., F.E.H., and A.O. prepared experimental substrates and samples. I.V.C. 508 calibrated the $\mathrm{Ca}^{2+}$ concentrations. T.F.B. designed, built, developed software for, and 509 characterized the photonic force microscope. T.F.B., F.E.H., and A.O. conducted the biophysical 510 experiments. T.F.B. carried out the data fitting and analysis of statistical significance. T.F.B., I.V.C., 511 F.E.H., A.O, and A.J.H. wrote the paper.

512 Competing interests The authors declare no competing interests. 
bioRxiv preprint doi: https://doi.org/10.1101/503029; this version posted December 20, 2018. The copyright holder for this preprint (which was not certified by peer review) is the author/funder, who has granted bioRxiv a license to display the preprint in perpetuity. It is made available under aCC-BY-NC-ND 4.0 International license.

\section{$514 \quad$ Figure legends}

a

b

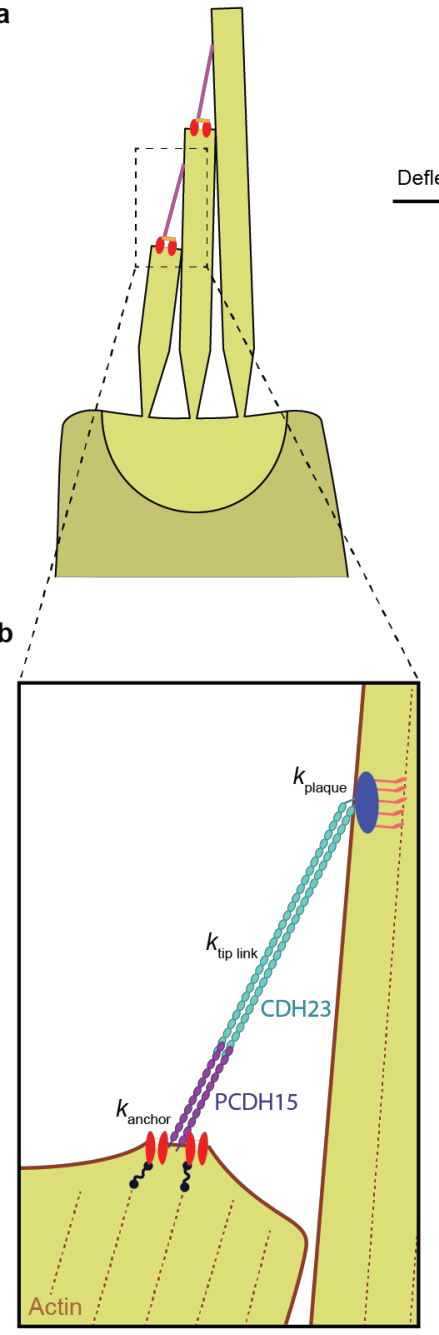

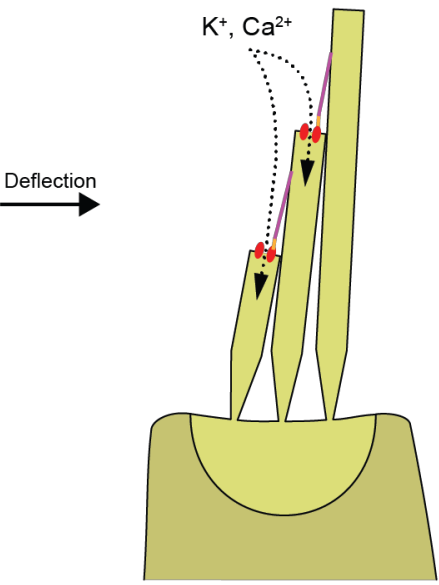

c

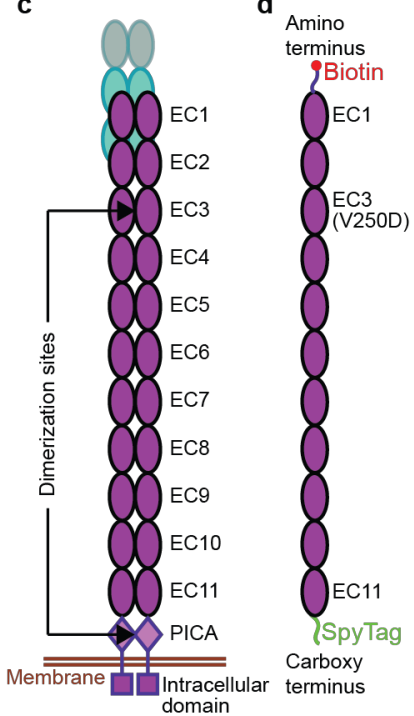

e

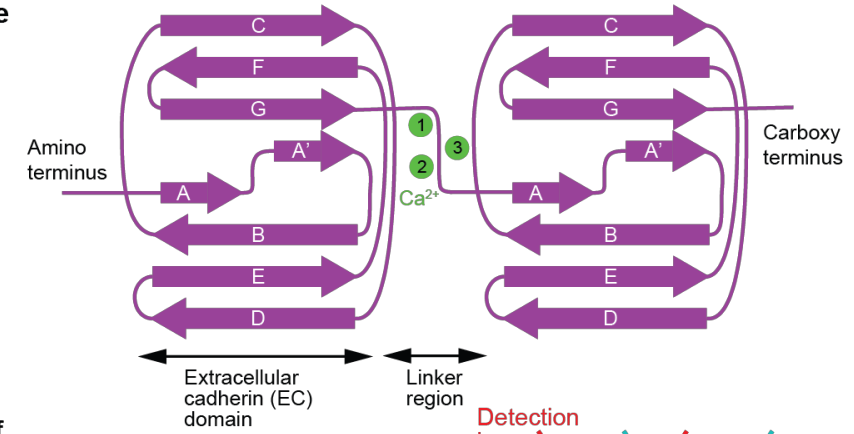

f

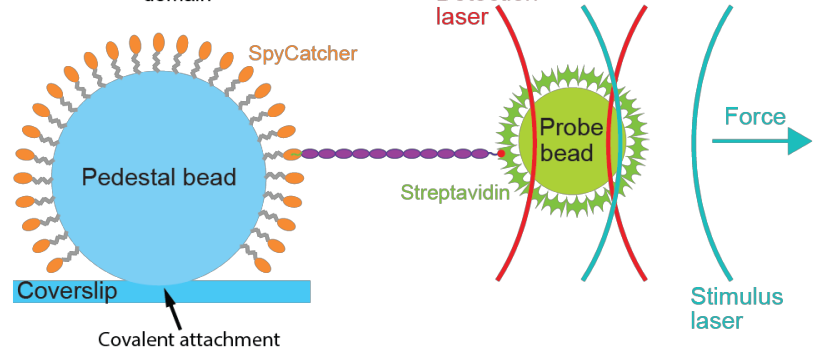


516 Fig. 1 | The role of tip-link proteins in transduction by hair cells. a, The hair bundle is a cluster

517 of stiff, actin-filled protrusions called stereocilia that stands atop each hair cell in the inner ear.

518 Each stereocilium is connected to its tallest adjacent neighbor through a proteinaceous filament

519 called a tip link (pink), which is coupled at its base to mechanically gated ion channels (red).

520 Deflection of a hair bundle increases the tension in the tip links, biasing the channels toward an

521 open state that allows the influx of positively charged ions. $\mathbf{b}$, The mechanical element that

522 converts hair-bundle displacement into a force capable of opening the channels is called the

523 gating spring. Its stiffness comprises those of the channel and its lower anchor ( $k_{\text {anchor }}$ ), the tip-

524 link proteins $\mathrm{PCDH} 15$ and $\mathrm{CDH} 23$ ( $\left.k_{\text {tip link }}\right)$, and the insertional plaque that anchors the link's top

525 end into the taller stereocilium ( $\left.k_{\text {plaque }}\right)$. c, The mechanical properties of the tip link emerge from

526 its quaternary structure and from the characteristics of its constituent proteins. The lower third

527 of the link consists of a dimer of PCDH15 molecules, each of which includes eleven extracellular

528 cadherin (EC) domains. d, To measure the mechanical behavior of monomeric PCDH15, we

529 tagged each end with a distinct molecular handle. We eliminated dimerization by a point

530 mutation (V250D) in domain EC3 and by truncation of the PICA domain. e, The folding motifs of

531 individual EC domains influence the mechanical properties of the full-length protein. Up to three

532 calcium ions (green) can bind between domains. f, We probe the mechanics of a PCDH15

533 monomer by confining it through its handles between an immobile, $2 \mu \mathrm{m}$ glass pedestal bead

534 and a diffusive, $1 \mu \mathrm{m}$ plastic probe bead. To acquire each force-extension relation, we measure

535 the position of the probe bead with a detection laser while applying a force with a stimulus laser. 
bioRxiv preprint doi: https://doi.org/10.1101/503029; this version posted December 20, 2018. The copyright holder for this preprint (which was not certified by peer review) is the author/funder, who has granted bioRxiv a license to display the preprint in perpetuity. It is made available under aCC-BY-NC-ND 4.0 International license.
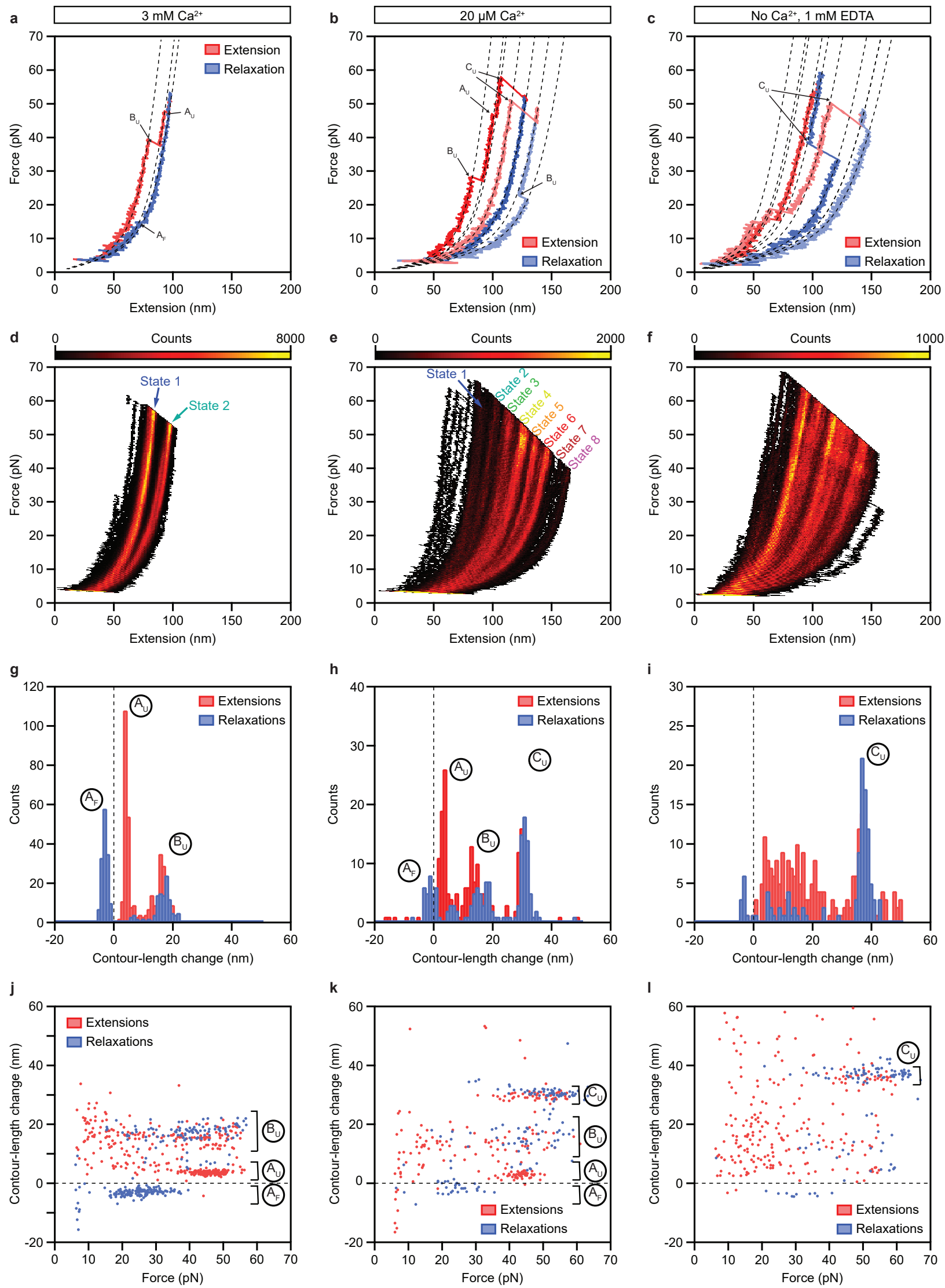
538 Fig. 2 | Force-extension measurements of PCDH15 monomers. a, At a $\mathrm{Ca}^{2+}$ concentration of

$5393 \mathrm{mM}$, individual force-extension cycles show two distinct classes of abrupt elongations, the

540 unfolding events $A_{U}$ and $B_{U}$, as well as refolding events of class $A_{F}$. The dashed lines represent fits

541 to the trajectories by a protein model. b, Reducing the $\mathrm{Ca}^{2+}$ concentration to $20 \mu \mathrm{M}$ elicits an

542 additional class of unfolding events, $C_{U}$, corresponding to the unfolding of entire cadherin

543 domains. $\mathrm{c}$, In the absence of $\mathrm{Ca}^{2+}$, unclassifiable structural changes occur in conjunction with the

544 well-defined events $C_{U}$. d-f, A heatmap displays all the force-extension cycles for a single

545 representative molecule at each $\mathrm{Ca}^{2+}$ concentration. The data were binned into pixels of

$5461 \mathrm{~nm} \times 0.1 \mathrm{pN}$. A much smaller portion of the state space is accessible for $3 \mathrm{mM}\left[\mathrm{Ca}^{2+}\right.$ than for

$54720 \mu \mathrm{M}\left[\mathrm{Ca}^{2+}\right]$ or in the absence of $\mathrm{Ca}^{2+} \cdot \mathbf{g}-\mathbf{i}$, Histograms of the contour-length changes of all abrupt

548 elongations verify that these rips can be grouped into classes $A_{F}, A_{U}, B_{U}$, and $C_{U}$ at $\mathrm{Ca}^{2+}$

549 concentrations of $3 \mathrm{mM}$ and $20 \mu \mathrm{M}$. In the absence of $\mathrm{Ca}^{2+}$, most of the contour-length changes

550 are more broadly distributed. $\mathrm{j}-\mathrm{I}$, Plots of the contour-length change of every rip against the force

551 at which that event occurred reveal the force distributions of each class of structural change.

552 Note that the extensions never completely unfolded a PCDH15 molecule, so elongations could

553 occur even during the relaxation phases. All force-extension cycles were sampled at intervals of

$55410 \mu \mathrm{s}$ and smoothed to a temporal resolution of $1 \mathrm{~ms}$. The waiting times between cycles were

$5550.2 \mathrm{~s}$ for $3 \mathrm{mM}\left[\mathrm{Ca}^{2+}\right], 2 \mathrm{~s}$ for $20 \mu \mathrm{M}\left[\mathrm{Ca}^{2+}\right]$, and $4 \mathrm{~s}$ in the absence of $\mathrm{Ca}^{2+}$. The number of cycles

556 recorded was 500 for $3 \mathrm{mM}\left[\mathrm{Ca}^{2+}\right]$ and 200 for $20 \mu \mathrm{M}\left[\mathrm{Ca}^{2+}\right]$ and in the absence of $\mathrm{Ca}^{2+}$. 


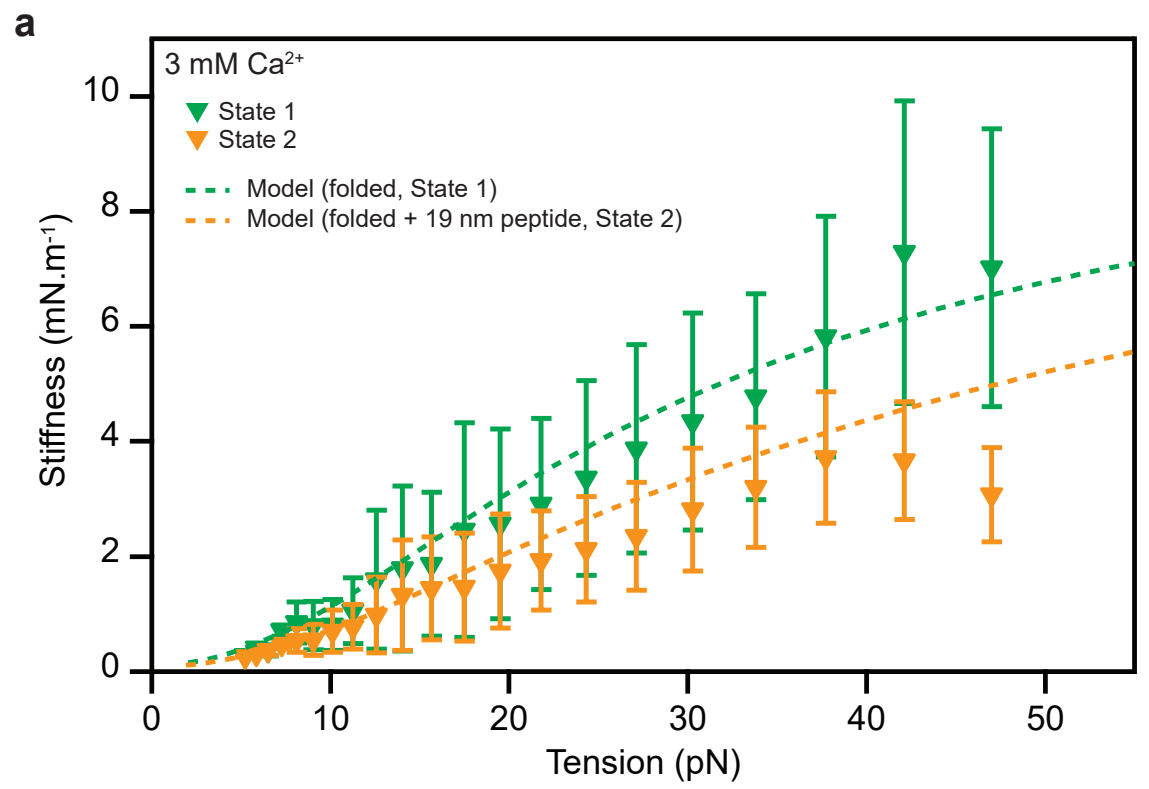

b

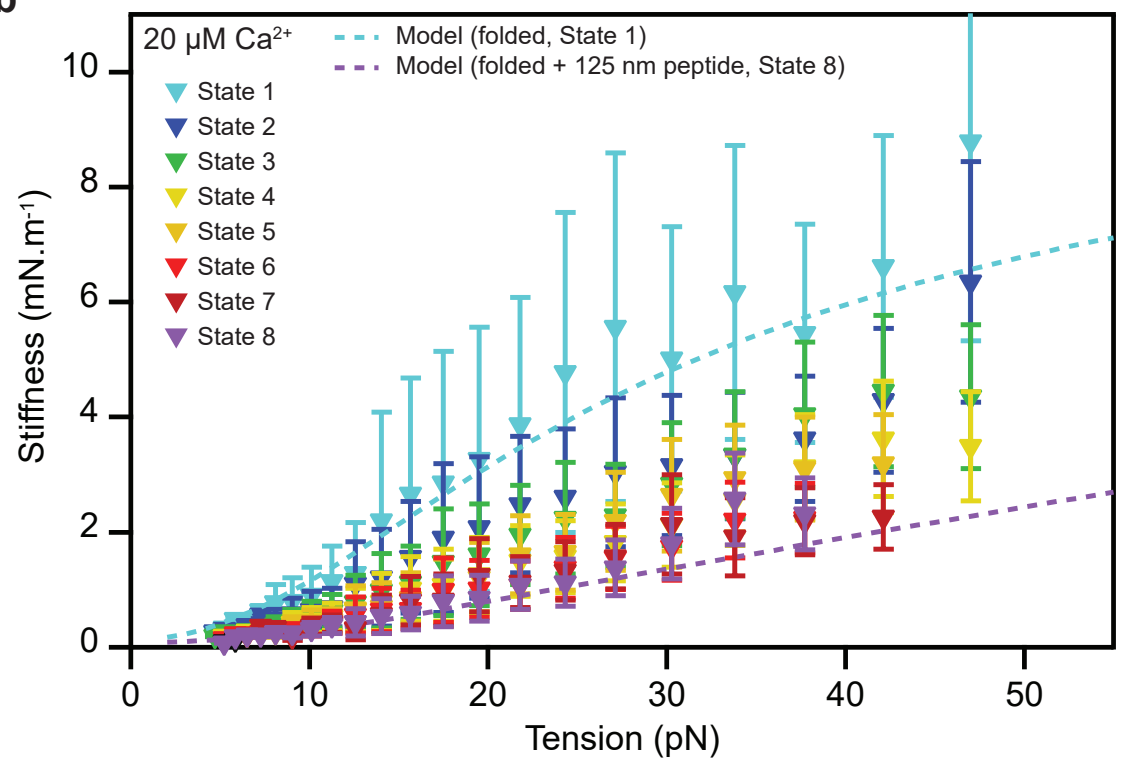

559 Fig. 3 | Stiffness of monomeric PCDH15. a, The stiffnesses of the different conformational states

560 of $\mathrm{PCDH} 15$ at a $\mathrm{Ca}^{2+}$ concentration of $3 \mathrm{mM}$ correspond to the slopes of the highly occupied

561 regions of the state space in Figure $2 \mathrm{~d}$,e and are corrected for the stiffness of the molecular tags

562 and anchors. The green dashed line represents the stiffness of our model of State 1, the fully

563 folded protein, with the parameter values of Table $1 \quad\left(b=3.0 \mathrm{~nm}, \quad / c_{\text {linker }}=1.35 \mathrm{~nm}\right.$,

$564 k_{\text {folded }}=10 \mathrm{mN} \cdot \mathrm{m}^{-1} ;$ parameter values were averaged over both $\mathrm{Ca}^{2+}$ concentrations). The orange

565 dashed line represents the model for State 2, with an additional $19 \mathrm{~nm}$ segment of unfolded 
566 protein with a persistence length of $0.49 \mathrm{~nm}$ representing the combined effect of the events $A_{U}$

567 and $\mathrm{Bu}$. b, The corresponding data for a $\mathrm{Ca}^{2+}$ concentration of $20 \mu \mathrm{M}$ capture a variety of

568 unfolding events leading to States 2-8. The light-blue dashed line represents the fully folded

569 protein (State 1); the purple dashed line depicts the stiffness of the protein in State 8, with an

570 unstructured peptide $125 \mathrm{~nm}$ in length to represent the unfolding of three cadherin domains in

571 series with contour-length changes of $15 \mathrm{~nm}$ and $4 \mathrm{~nm}$. The experimental data are means \pm SEMs

572 for five molecules and six molecules at a $\mathrm{Ca}^{2+}$ concentration of respectively $3 \mathrm{mM}$ and $20 \mu \mathrm{M}$. 


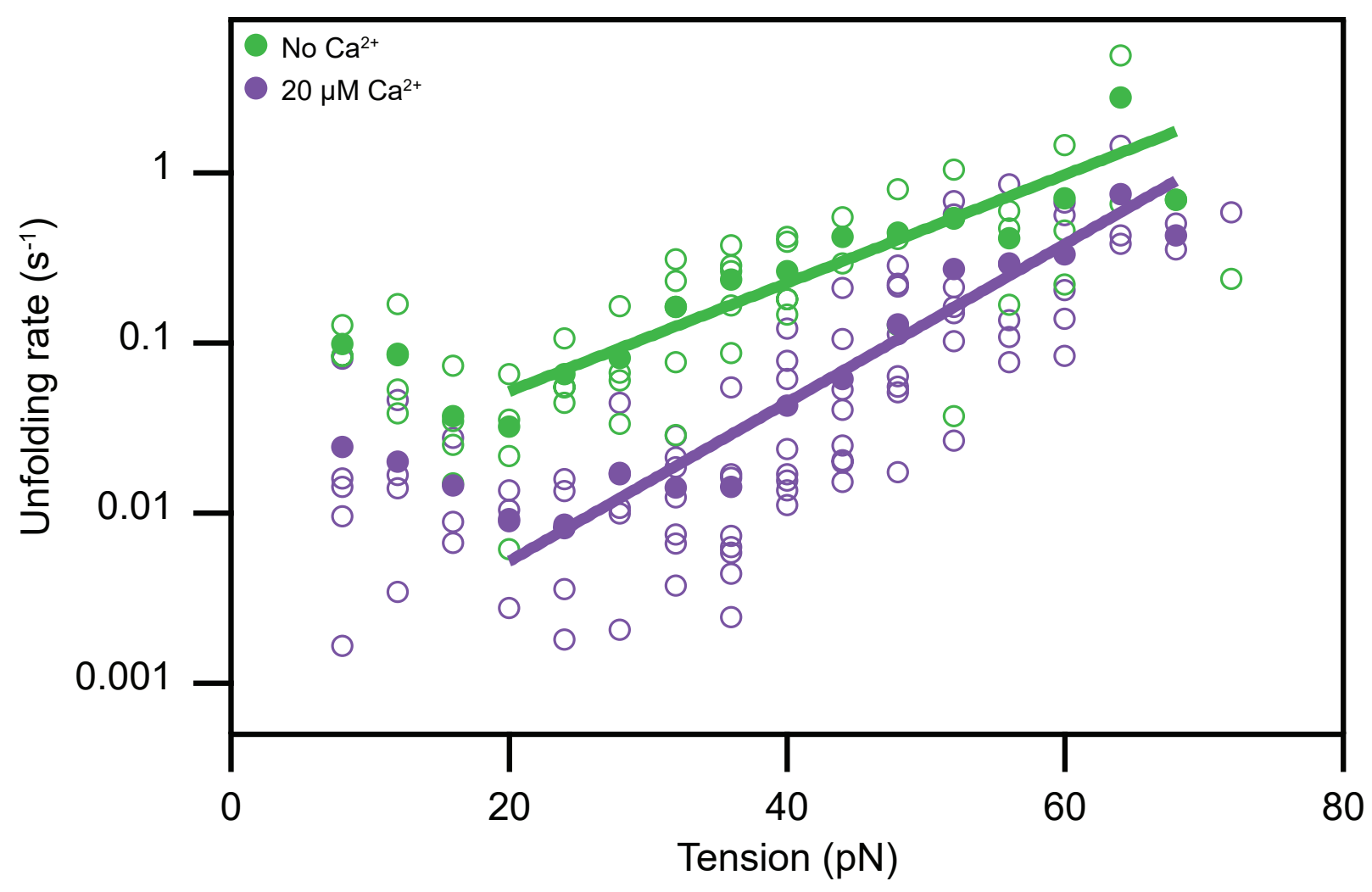

575 Fig. 4 | Tension-dependent unfolding rates of a single cadherin domain. a, Assuming that all

576 domains of PCDH15 are similar and unfold independently, we estimate the rate at which 577 individual EC domains unfold as a function of tension. Domains unfold much more readily in the 578 absence of $\mathrm{Ca}^{2+}$ (green) than at a physiological $\mathrm{Ca}^{2+}$ concentration of $20 \mu \mathrm{M}$ (purple). The filled 579 circles represent the means for eight molecules at $20 \mu \mathrm{M}\left[\mathrm{Ca}^{2+}\right]$ and for five molecules in the 580 absence of $\mathrm{Ca}^{2+}$. The outlined circles, which represent the data for individual molecules, provide 581 an estimate of the data's spread. The solid lines are fits of Bells' model ${ }^{44}$ to the data: for $20 \mu \mathrm{M}$ $582 \mathrm{Ca}^{2+}$, the unfolding rate at zero force is $k_{0}=0.0006 \pm 0.0002 \mathrm{~s}^{-1}$ and the transition-state distance 583 is $x^{\ddagger}=0.44 \pm 0.04 \mathrm{~nm}$; in the absence of $\mathrm{Ca}^{2+}, k_{0}=0.012 \pm 0.006 \mathrm{~s}^{-1}$ and $x^{\ddagger}=0.30 \pm 0.04 \mathrm{~nm}$. 
a

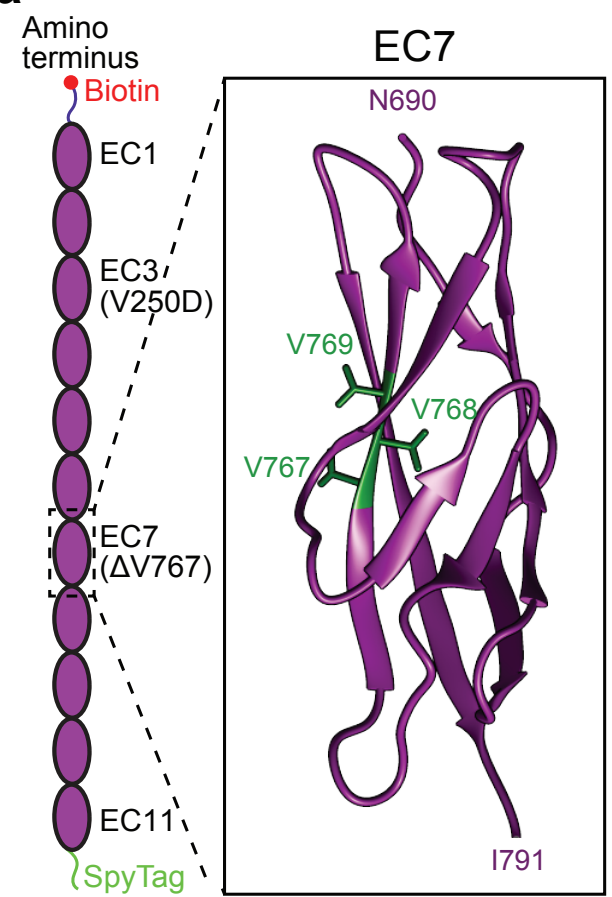

Carboxy

terminus b

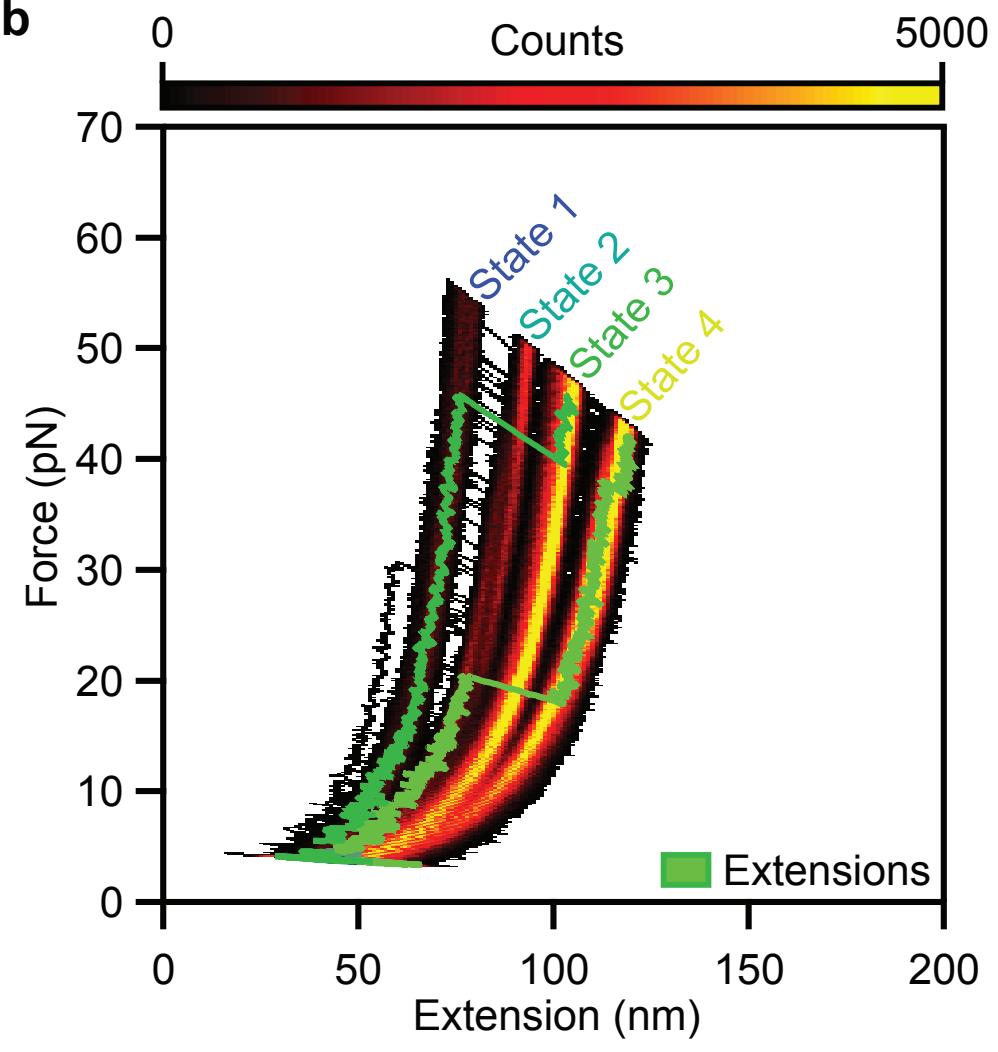

Fig. 5 | Effect of a hearing loss-associated mutation on PCDH15 mechanics. a, We deleted valine

587767 in the seventh EC domain of PCDH15. As indicated in the crystal structure (PDB ID code 588 5W1D, image generated with UCSF Chimera), V767 is located in the F strand of the cadherin fold.

589 b, A state-space heatmap for 500 extension-relaxation cycles reveals that at $3 \mathrm{mM}\left[\mathrm{Ca}^{2+}\right]$ the 590 mutant protein can assume four distinct conformational states. The two additional states not 591 observed in the wild-type protein result from unfolding of the pathologic cadherin domain in 592 series with the usual States 1 and 2. Unfolding of the pathological domain is rare and occurs in 593 only a few cycles, two of which are superimposed upon the heat map (green traces). The waiting 594 time between cycles was $0.2 \mathrm{~s}$. 
Table

597 Table 1 - Material property values of PCDH15.

\begin{tabular}{|c|c|c|c|c|c|c|}
\hline$\left[\mathrm{Ca}^{2+}\right]$ & $b(\mathrm{~nm})$ & $I$ Clinker $(\mathrm{nm})$ & $k_{\text {folded }}(\mathrm{mN} / \mathrm{m})$ & $\Delta / \mathrm{C}_{\mathrm{A}}(\mathrm{nm})$ & $\Delta / \mathrm{c}_{\mathrm{B}}(\mathrm{nm})$ & $\Delta / \mathrm{c}_{\mathrm{C}}(\mathrm{nm})$ \\
\hline $3 \mathrm{mM}$ & $2.9 \pm 0.5$ & $1.4 \pm 0.5$ & $9 \pm 4$ & $4.0 \pm 0.2$ & $15.8 \pm 0.7$ & - \\
\hline $20 \mu \mathrm{M}$ & $3.1 \pm 0.4$ & $1.3 \pm 0.5$ & $11 \pm 5$ & $3.9 \pm 0.4$ & $15.0 \pm 0.6$ & $35 \pm 1$ \\
\hline
\end{tabular}

598 Measurements are given as means \pm SEMs for five experiments with $3 \mathrm{mM}\left[\mathrm{Ca}^{2+}\right]$ and for eight

599 experiments with $20 \mu \mathrm{M}\left[\mathrm{Ca}^{2+}\right]$.

600 


\section{Methods}

\section{Molecular cloning}

603 Plasmids were assembled by Gibson assembly in a one-step isothermal reaction using home604 made master mixes ${ }^{45}$. We assembled in a pLEXm backbone ${ }^{46}$ a construct encoding the protein 605 signal peptide-QYDDDWQYED-Avitag-GSGSGS-PCDH15(EC1-11, V250D)-GSGSGS-Spytag-6xHis. 606 The deletion mutant (V250D, $\triangle$ V767) was assembled in a similar reaction. The PCDH15 sequence 607 was isoform 1 from Mus musculus (UniProtKB entry Q99PJ1). The signal peptide comprises the 608 native sequence that leads to secretion of PCDH15. Two tags for the site-specific confinement of 609 PCDH15 were fused to the termini of the protein. The Avitag, with the sequence 610 GLNDIFEAQKIEWHE, is recognized by a biotin ligase (BirA-500, Avidity, Aurora, CO, USA), which 611 covalently biotinylates the lysine side chain. The SpyTag had the sequence AHIVMVDAYKPTK. 612 The tags were attached to PCDH15 through flexible GSGSGS linkers.

\section{Expression of recombinant PCDH15}

614 All constructs were transfected with 40 kDa polyethyleneimine (Polysciences, Inc., Warrington, 615 PA, USA) into suspension-adapted HEK293 cells (Freestyle, R79007, Thermo Fisher Scientific, 616 Waltham, MA, USA). Seven to nine days post transfection, the medium was collected and 617 secreted proteins were purified by $\mathrm{Ni}^{2+}$-affinity chromatography. Proteins were further purified 618 by size-exclusion chromatography (Superose 6 10/300 GL,17517201, GE Healthcare Bio-Sciences, 619 Pittsburgh, PA, USA) in $10 \mathrm{mM}$ Tris, $150 \mathrm{mM} \mathrm{NaCl}$, and $3 \mathrm{mM} \mathrm{CaCl}_{2}$. The purified proteins were 620 concentrated to $1.5 \mathrm{mg} / \mathrm{ml}$ and biotinylated for $1 \mathrm{hr}$ at $30^{\circ} \mathrm{C}$ with biotin ligase (BirA 500, Avidity, 621 Aurora, CO, USA). The samples were then used immediately or mixed with equal volumes of 622 glycerol and stored at $-20^{\circ} \mathrm{C}$ for up to four months.

623 Design of the control linker peptide

624 In order to test the mechanical properties of the linkers and anchors of the assay in the absence 625 of PCDH15, we fused the SpyTag and AviTag by a flexible GGGSGGGS linker to produce a control 
626 linker peptide with the sequence AHIVMVDAYKPTKGGGSGGGSGLNDIFEAQKIEWHE (Genscript,

627 Piscataway, NJ, USA). After biotinylation, this peptide is capable of tethering streptavidin-coated 628 probe beads to SpyCatcher molecules on pedestal beads, representing a control single-molecule 629 assay that contains all components except for the PCDH15 protein.

630 Site-specific attachment of PCDH15 through short molecular anchors

631 The carboxy terminus of PCDH15 was modified with a SpyTag capable of forming a covalent bond

632 with a small, globular protein called SpyCatcher, which was linked to the surface of the pedestal

633 bead. The protein's amino terminus was biotinylated to allow its strong, site-specific attachment

634 to streptavidin molecules on the probe bead (Figure 1d,f). The SpyCatcher-SpyTag complex is 635 mechanically stable up to nanonewtowns of force, well beyond the range relevant for hearing ${ }^{47}$. 636 The streptavidin-biotin interaction has a lifetime of about $30 \mathrm{~s}$ for the highest forces applied in 637 this work ${ }^{16,48}$. These properties made this set of tags and binding partners ideal for the site638 specific confinement of proteins under force.

639 Conjugation of pedestal beads with SpyCatcher molecules

640 Cys-SpyCatcher molecules (EOX004, Kerafast, Boston, MA, USA) were conjugated to aminated 641 silicon dioxide microspheres (140414-10, Corpuscular, Cold Spring, New York, USA) through 642 short, bifunctional polyethylene glycol spacers. To deprotonate the surface amine groups, $200 \mu \mathrm{l}$ 643 of the beads was washed once and resuspended for $1 \mathrm{hr}$ at room temperature in $100 \mu \mathrm{l}$ of $50 \mathrm{mM}$ 644 sodium tetraborate buffer (11625, Sigma Aldrich, St Louis, MO, USA) at pH 8.5. Deprotonation is 645 necessary for efficient covalent coupling of the $N$-hydroxysuccinimide-PEG 12 -maleimide spacer 646 (22112, ThermoFisher, Waltham, Ma, USA), which we added to the beads to a final concentration 647 of $50 \mathrm{mM}$ of the linker and a final volume of $160 \mu \mathrm{l}$. The resulting mixture was incubated for $64830 \mathrm{~min}$ at room temperature, washed three times with $1 \mathrm{ml}$ of Hepes-buffered saline solution 649 (HeBS; $20 \mathrm{mM}$ Hepes and $100 \mathrm{mM} \mathrm{NaCl}$ ), and after the final wash resuspended into $250 \mu \mathrm{l}$ of 650 HeBS. 
Meanwhile, $0.5 \mathrm{mg}$ of Cys-SpyCatcher protein was dissolved in $50 \mu \mathrm{l}$ of HeBS and reduced

652 for $1 \mathrm{hr}$ with tris(2-carboxyethyl)phosphine (Immobilized TCEP Disulfide Reducing Gel, 77712,

653 ThermoFisher Scientific, Waltham, MA, USA) according to the manufacturer's instructions. The

654 reduced SpyCatcher protein was then mixed with $55 \mu \mathrm{l}$ of $100 \mathrm{mg} / \mathrm{ml}$ sulfhydryl-blocked bovine 655 serum albumin (BSA; 100-10SB, Lee Biosolutions, Inc., Maryland Heights, MO, USA) in HeBS. The 656 bead solution was injected into the protein mixture and incubated at $4{ }^{\circ} \mathrm{C}$ overnight to allow the 657 covalent attachment of the SpyCatchers' unique cysteines to the maleimide residues on the 658 pedestal bead. The beads were then washed three times with $1 \mathrm{ml}$ of HeBS and resuspended to 659 a volume of $100 \mu \mathrm{l}$ in HeBS. Any unreacted maleimide was quenched by addition of $100 \mu \mathrm{l}$ of $1 \mathrm{M}$ 660 L-cysteine (11033-016, Gibco BRL, Gaithersburg, MD, USA) in HeBS and incubation for $1 \mathrm{hr}$. 661 Finally, the beads were washed three times and stored at $4^{\circ} \mathrm{C}$ in HeBS with $0.02 \%$ sodium azide 662 (71289, Sigma-Aldrich, St. Louis, MO, USA).

663 Covalent attachment of pedestal beads

664 We covalently attached pedestal beads through the surface amine groups of their SpyCatcher 665 molecules to $\mathrm{COOH}$-modified glass coverslips through carbodiimide crosslinking. Glass coverslips 666 (12-545-81, Thermo Fisher Scientific, Waltham, MA, USA) were cleaned by sonication in ethanol 667 for $15 \mathrm{~min}$. After drying under a stream of oxygen, the coverslips were transferred to a solution 668 of $1.5 \mathrm{~g}$ Nochromix (Godax Laboratories, Cabin John, MD, USA) in $60 \mathrm{ml}$ sulfuric acid (A300S-500, 669 Thermo Fisher Scientific, Waltham, MA, USA) and incubated for $3 \mathrm{hr}$. We then washed the 670 coverslips three times with deionized water followed by three washes with ethanol. During each 671 wash the coverslips were sonicated for five minutes in a bath sonicator. The slides were dried 672 under a stream of oxygen gas and oxidized for $30 \mathrm{~min}$ in an ultraviolet ozone cleaner (PC440, 673 Bioforce Nanosciences, Salt Lake City, UT, USA). In this step oxidation occurred on only one side 674 of the coverslips, the "functional" side, which was used in all the subsequent steps. We vapor-deposited an aminosilane layer onto the oxidized glass surfaces by placing the 676 coverslips into a gas-tight glass container together with-but not submerged in-100 $\mu$ of 
677 (3-aminopropyl)trimethoxysilane (281778, Sigma Aldrich, Inc, St Louis, MO, USA) dissolved in

$67810 \mathrm{ml}$ of toluene (T324-1, Thermo Fisher Scientific, Waltham, MA, USA) ${ }^{49}$. The container was

679 incubated overnight at $80^{\circ} \mathrm{C}$. Following the vapor deposition, the coverslips were washed three

680 times in ethanol, dried, and placed into a solution of $50 \mathrm{mg}$ succinic anhydride (S7626, Sigma

681 Aldrich, St Louis, MO, USA) dissolved in $1 \mathrm{ml}$ dimethyl sulfoxide (D4540, Sigma Aldrich, St Louis,

$682 \mathrm{MO}, \mathrm{USA}$ ) and left to incubate at room temperature for at least $3 \mathrm{hr}$. This step converted the

683 vapor-deposited amine groups into carboxyl groups and rendered the surface suitable for

684 coupling. The carboxylated slides were then rinsed three times with ethanol and either used

685 immediately or stored in ethanol for up to $72 \mathrm{hr}$.

Assembly of samples

687 To assemble a sample, a carboxylated coverslip was dried and secured with vacuum grease onto 688 a metal washer, so that its functional side contacted the washer (Extended Data Fig. 7). (1-ethyl-

689 3-(3-dimethylaminopropyl)carbodiimide and $\mathrm{N}$-hydroxysulfosuccinimide (respectively 77149 and

690 24510, Thermo Fisher Scientific, Waltham, MA, USA) were equilibrated to room temperature

691 before $10 \mathrm{mg}$ of each reagent was dissolved in $1 \mathrm{ml}$ of activation-buffer solution containing

$69210 \mathrm{mM} \mathrm{NaCl}$ and $1 \mathrm{mM}$ 2-(N-morpholino)ethanesulfonic acid (M3671, Sigma Aldrich, St Louis,

$693 \mathrm{MO}, \mathrm{USA})$ at $\mathrm{pH}$ 6. Of the resulting solution, $50 \mu \mathrm{l}$ was pipetted onto the functional surface of the

694 mounted slide and left to incubate at room temperature for exactly $30 \mathrm{~min}$ to activate the slides

695 with amine-reactive hydroxysulfosuccinimide esters. To remove any excess reagents, we

696 carefully washed the activated slide by pipetting $2 \mathrm{ml}$ of Hepes-buffered saline solution with $\mathrm{Ca}^{2+}$

697 (HeBS-Ca, $20 \mathrm{mM}$ Hepes, $100 \mathrm{mM} \mathrm{NaCl}, 1 \mathrm{mM} \mathrm{CaCl}_{2}$ ) onto the mounted coverslip. Immediately

698 after removing the solution except for a thin film to keep the active surface from drying, we

699 pipetted pedestal beads in HeBS-Ca onto the activated surface and allowed to react for $2 \mathrm{hr}$.

700 Mounting a second coverslip on the top of the washer closed the sample chamber. Access ports

701 in the washer allowed the exchange of solutions within the chamber. To reduce non-specific

702 interactions of PCDH15 with the beads, we exchanged the fluid to blocking-buffer solution 
containing $10 \mathrm{mg} / \mathrm{ml}$ sulfhydryl-blocked bovine serum albumin (100-10SB, Lee Biosolutions, Inc.,

704 Maryland Heights, MO, USA), $150 \mathrm{mM} \mathrm{NaCl}, 20 \mathrm{mM}$ Tris- $\mathrm{HCl}$, and $3 \mathrm{mM} \mathrm{CaCl}_{2}$ at $\mathrm{pH}$ 8. The sample was stored overnight at $4{ }^{\circ} \mathrm{C}$ before addition of the PCDH15 molecules.

PCDH15 monomers were diluted into blocking-buffer solution and flushed into a sample chamber. The molecules were sufficiently dilute to ensure that any tether was likely with an average probability exceeding $90 \%$ to represent a single molecule of PCDH15, rather than two or more at once (Extended Data Table 1). We incubated the sample for $1 \mathrm{hr}$ at room temperature

711 to allow the carboxy termini of the PCDH15 molecules, each fused to a SpyTag, to covalently bind 712 to the SpyCatcher proteins on the surface of the pedestal beads. The biotinylated amino termini

713 were then directed radially outward from the pedestal beads and thus available for coupling to 714 streptavidin-coated probe beads. The sample was subsequently washed with copious amounts 715 of blocking-buffer solution to remove any unbound PCDH15 monomers.

716 The blocking buffer was replaced with sample-buffer solution consisting of $20 \mathrm{mM}$ Tris$717 \mathrm{HCl} \mathrm{pH} \mathrm{7.5,} 150 \mathrm{mM} \mathrm{NaCl}$, and $10 \mathrm{mg} / \mathrm{ml}$ sulfhydryl-blocked bovine serum albumin (100-10SB, 718 Lee Biosolutions, Inc., Maryland Heights, MO, USA). The solution contained probe beads 719 (CP01004, Bangs Laboratories, Fishers, IN, USA); depending on the experiment, it included 3 mM $720 \mathrm{CaCl}_{2}, 20 \mu \mathrm{M} \mathrm{CaCl}_{2}$, or $1 \mathrm{mM}$ EDTA. To protect PCDH15 from photodamage, we employed an 721 oxygen-scavenging system consisting of 18 mM D-glucose (G-5400, Sigma-Aldrich, St. Louis, MO, 722 USA), $13 \mathrm{U} / \mathrm{ml}$ pyranose oxidase (P4234, Sigma Aldrich, St Louis, MO, USA), and $8500 \mathrm{U} / \mathrm{ml}$ 723 catalase (219261, Millipore Sigma, Burlington, MA, USA) $)^{50,51}$.

\section{Calibration of $\mathrm{Ca}^{2+}$ concentration}

725 We used a fluorescence assay to confirm that the binding of $\mathrm{Ca}^{2+}$ to BSA does not significantly 726 alter the concentration of free $\mathrm{Ca}^{2+}$ in sample-buffer solution (Extended Data Fig. 8). Using $3 \mu \mathrm{M}$ 727 of the fluorescent calcium indicator Fluo-5N (F14203, ThermoFisher Scientific, Waltham, MA, 728 USA), we tested the fluorescence of the solution with and without $10 \mathrm{mg} / \mathrm{ml}$ BSA at various total 
$729 \mathrm{Ca}^{2+}$ concentrations. Our data show that BSA at this concentration does not noticeably change

730 the concentration of free $\mathrm{Ca}^{2+}$, whereas a very high BSA concentration, $100 \mathrm{mg} / \mathrm{ml}$, sequesters a

731 significant amount of $\mathrm{Ca}^{2+}$ (Extended Data Fig. 8b).

732 High-bandwidth and high-precision optical trapping and tracking

733 All data were acquired using a custom-built photonic-force microscope ${ }^{22}$, which in this instance

734 was upright rather than inverted. The microscope could track the three-dimensional position of

735 a weakly optically trapped, $1 \mu \mathrm{m}$-diameter probe bead with an integration time of $1 \mu \mathrm{s}$, sampled

736 at a frequency of $100 \mathrm{kHz}$, with sub-nanometer precision. In brief, the position-sensing $1064 \mathrm{~nm}$

737 laser beam (Mephisto $500 \mathrm{~mW}$, Coherent, CA, USA) was expanded and focused into the sample

738 chamber through a high-numerical aperture water-immersion objective lens (UPlanSApo 60xW,

739 Olympus, Tokyo, Japan). This beam formed a weak optical trap that confined the probe bead. We

740 collected light forward-scattered by the probe bead together with the unscattered portion of the

741 beam on a quadrant photodiode, where the two waves interfered. The signals of the four

742 quadrants were related to the three-dimensional position of the bead in the optical trap ${ }^{21}$. The

743 microscope's position error over one extension-relaxation cycle with a duration of $1 \mathrm{~s}$ was given

744 by its position noise from $1 \mathrm{~Hz}$ to $1 \mathrm{MHz}$, for which we measured root-mean-square values of

$7450.6 \mathrm{~nm}, 0.3 \mathrm{~nm}$, and $0.4 \mathrm{~nm}$ along the $x_{-}, y_{-}$, and $z$-axes respectively ${ }^{52,53}$. Experiments were

746 performed with the protein tether oriented along the $y$-axis, that with highest precision. Typical

747 spring constants of the weak position-sensing trap were $6 \mu \mathrm{N} \cdot \mathrm{m}^{-1}, 7.5 \mu \mathrm{N} \cdot \mathrm{m}^{-1}$, and $2 \mu \mathrm{N} \cdot \mathrm{m}^{-1}$ along

748 the $x_{-}^{-}, y^{-}$, and $z$-axes respectively. At very high stimulus forces of tens of piconewtons the probe

749 bead was at its maximum extension of about $150 \mathrm{~nm}$ along the $\mathrm{y}$-axis from the center of the 750 position-sensing optical trap, corresponding to a maximum force generated by this trap of $1.1 \mathrm{pN}$.

751 For lower stimulus forces and smaller extensions, the force was considerably less than $1 \mathrm{pN}$.

752 Because the force generated by the position-sensing optical trap was very small compared to the 753 stimulus force, we disregarded it in our analysis. 
To record hundreds of cycles, we required observation times greater than a few seconds and therefore had to compensate the microscope's slight mechanical drift. The position of the sample relative to the optical traps was controlled by a nano-positioning stage (NanoView/M375HS, Mad City Labs, WI, USA), whose position we adjusted to compensate for the drift (see below). With drift compensation in effect, the root-mean-square deviation measured along the $\mathrm{y}$-axis between $\mathrm{DC}$ and $1 \mathrm{MHz}$ during 5 min of observation was $2 \mathrm{~nm}$.

In addition to the previously described position-sensing weak optical trap, we added a second optical trap to the system to apply force stimuli to the tethered proteins. We chose a wavelength of $852 \mathrm{~nm}$ (DL852-500, Crystalaser, Reno, NV, USA), which is near a local minimum of the action spectrum of photodamage to biological material ${ }^{54}$. Using a beam-steering lens mounted on a three-dimensional piezoelectric-block translator (P.282.30, Physik Instrumente, Auburn, MA, USA), we could shift the position of the stimulus trap with respect to the positionsensing trap with nanometer precision. Over $18 \mathrm{~min}$ of observation time, the average drift of the two optical traps relative to each other was $3 \mathrm{pm} \cdot \mathrm{s}^{-1}$. The $852 \mathrm{~nm}$ laser beam traversed an electrooptical modulator (LM13, Excelitas Technologies, Fremont, CA, USA) placed between crossed polarizing beam-splitting cubes, which allowed us to modulate the intensity and thus the stiffness of the stimulus trap.

\section{Drift compensation}

772 Although we designed the microscope's frame to minimize thermal drifts of the sample chamber 773 with respect to the optical traps, a small drift of about $250 \mathrm{pm} \cdot \mathrm{s}^{-1}$ remained $^{22}$. To eliminate this

774 drift, we implemented an active-feedback mechanism by using as a fiducial marker a second 775 pedestal bead situated tens of micrometers from the pedestal to which a single molecule was 776 tethered. The motion of the pedestal bead reflects the drift of the sample chamber with respect 777 to the microscope frame. A camera (pco.edge 5.5, PCO, Kelheim, Germany) tracked the threedimensional position of this pedestal bead at a frequency of $5 \mathrm{~Hz}$. The pedestal's position signal 
780 position of the nano-positioning stage to compensate for the sample's drift. To test the fidelity

781 of this method, we immobilized a probe bead on a sample chamber's coverslip, positioned it at

782 the focus of the position-sensing optical trap, and used the microscope to determine its position

783 while drift compensation was active. We found that linear drifts were eliminated and that the

784 root-mean-square variation of the position signal along the $y$-axis of $2 \mathrm{~nm}$ over 5 min remained.

785 Calibration, linearization, and correction of the probe-position signal

786 The three-dimensional detector's non-linear response was linearized and calibrated in situ for

787 each individual probe bead ${ }^{55}$. The calibration depended upon the viscosity of the buffer solution,

788 which we corrected for the presence of $10 \mathrm{mg} / \mathrm{ml}$ bovine serum albumin as described ${ }^{56}$.

789 The probe bead forward-scattered a portion of the position-sensing laser beam, and we

790 detected the bead's three-dimensional position by monitoring the interference pattern of the

791 beam's scattered and unscattered light wave on a quadrant photodiode ${ }^{21}$. Scattering of a small

792 amount of the beam by the pedestal bead produced an artifact in the signal ${ }^{22}$ that was eliminated

793 by the subtraction of a reference signal.

794 Calibration and correction of the stimulus trap

795 Before each single-molecule experiment and for each individual probe bead, we measured the

796 relation between the intensity of the stimulus laser and the stiffness of the stimulus trap. While

797 monitoring the Brownian motion of the trapped probe bead with the position-sensing optical

798 trap, we increased the power of the stimulus beam in $10 \%$ increments. Fitting of the position

799 signal's power spectral density for each intensity with a hydrodynamically correct theory ${ }^{57}$

800 yielded the stiffness as a linear function of intensity. During our experiments, we sampled the

801 intensity of the stimulus laser-and hence the trap's stiffness - with the same sampling rate as

802 the position signal of the probe bead.

803 We adjusted the intensity of the stimulus laser using an electro-optical modulator. At high

804 attenuation, close to extinction, the beam profile at the exit of the modulator deviated from a

805 Gaussian function, which laterally shifted the position of the stimulus trap within the sample 
806 chamber by a few nanometers. We recorded this shift during the calibration procedure for each

807 probe bead and accounted for it when calculating the laser intensity-dependent force on the 808 probe bead.

810 To initiate a single-molecule experiment, we optically trapped a probe bead deep in solution and

811 calibrated the position sensor and the stiffnesses of both optical traps. We next positioned the

812 bead's center at a height of $1 \mu \mathrm{m}$ from the functionalized coverslip, with its mean axial position

813 at the equator of the attached pedestal beads. This height was determined by slowly moving the

814 coverslip towards the optical trap until the probe bead's axial thermal motion began to be

815 confined by the coverslip, then retracting the coverslip by an appropriate distance ${ }^{52}$.

816 The sample was moved laterally so that the probe bead was aligned along the $y$-axis with

817 a pedestal bead. After recording a reference signal to account for light scattered by the pedestal

818 we gently maneuvered the pedestal bead towards the optical trap until the probe's Brownian

819 motion along the $y$-axis began to be restricted by the pedestal. When a PCDH15 molecule was

820 present on the surface of the pedestal bead and within reach of the Brownian motion of the

821 probe bead, a single molecule tether formed between the amino terminal biotin on the protein

822 and a streptavidin molecule on the probe bead. The concentration of PCDH15 was titrated so

823 that only a fraction of such approaches resulted in tether formation, on average giving rise to a

$82490 \%$ confidence of single-molecule conditions ${ }^{58}$ (Extended Data Table 1). The position of the

825 stimulus trap was then displaced by $200 \mathrm{~nm}$ along the $\mathrm{y}$-axis. Before the force was lowered to the

826 holding level, a brief increase in the intensity of the stimulus beam provided a force pulse of

827 several tens of piconewtons to the tethered protein. This operation ensured that no portion of

828 the protein was nonspecifically attached to either of the confining surfaces and that the full

829 contour of the protein linked the two beads at the beginning of the extension-relaxation cycles.

830 Force ramps were then applied to the tethered protein by repeatedly increasing and decreasing 831 the intensity of the stimulus trap (Extended Data Fig. 1). 


\section{Control of non-specific attachments}

833 For a successful single-molecule experiment it is imperative that the overwhelming majority of

834 tethers between pedestal and probe beads constitutes PCDH15 molecules anchored at their 835 amino and carboxy termini by respectively biotin and SpyTag: only a very small number of non836 specific tethers should occur.

We tested whether streptavidin-coated probe beads would tether to SpyCatcher 838 molecules on pedestal beads in the absence of PCDH15. Out of 65 attempts of initiating such 839 non-specific tethering with two samples and four probe beads, only one bond formed, which 840 ripped off immediately upon application of a stimulus force. We concluded that all stable tethers 841 that we observed in our single-molecule experiments resulted from PCDH15 molecules or, in the 842 case of control experiments to test the assay's mechanics, from linker-peptide constructs.

To exclude any non-specific interactions between PCDH15 and either of the beads, we 844 next confirmed that these PCDH15 tethers formed only if both the SpyTag-SpyCatcher and biotin845 streptavidin interactions were present. Pedestal beads were coupled to a coverslip and incubated 846 with $0.15 \mathrm{mg} / \mathrm{ml} \mathrm{PCDH15}$ in blocking-buffer solution for $1 \mathrm{hr}$ at room temperature to allow the 847 proteins to react with the pedestals. The coverslip was then washed with copious amounts of 848 blocking-buffer solution to remove any free PCDH15 molecules before the addition of a high 849 concentration of probe beads in blocking-buffer solution. The probe beads were allowed to bind 850 to the PCDH15 molecules on the pedestal beads for $1 \mathrm{hr}$ before the coverslip was washed once 851 more and then imaged.

852 As a positive control, with both sets of anchors intact, we found an average of 3.25 probe 853 beads bound to each pedestal bead for the given reaction conditions (Extended Data Table 2). To 854 test whether the carboxy-terminal SpyCatcher-SpyTag anchor participated in the tether, we 855 generated pedestal beads without SpyCatcher and attempted to attach probe beads to them 856 through $\mathrm{PCDH} 15$ molecules using the procedure described above. We found that tethering of 857 probe beads was completely abolished in the absence of SpyCatcher, confirming that the 
858 SpyCatcher-SpyTag interaction was an essential part of the formed tethers (Extended Data 859 Table 2).

To determine whether the amino termini of our tethers were anchored to the probe

861 beads through the biotin-streptavidin interaction, we attempted to tether probe beads to 862 SpyCatcher-positive pedestals through PCDH15 molecules that had not been biotinylated. We 863 again found that tether formation was completely abolished, confirming that the amino-terminal 864 confinement in our single-molecule experiments occurred through the biotin-streptavidin 865 interaction (Extended Data Table 2).

866 We concluded that our single-molecule assay was highly specific: molecular tethers 867 formed in the presence of an appropriately tagged construct. If either of the pairs of anchors was 868 disrupted, tether formation was completely abolished.

870 The anchor position of the protein tether along the axis of extension was an important parameter 871 that had to be determined before our protein model could be fit to the data. We determined this 872 anchor position by analyzing the three-dimensional probability distribution of the motion of the 873 tethered probe bead in the absence of externally applied tension. The pedestal bead appeared as

874 a forbidden volume in the spatial probability distribution of the tethered probe bead, a so-called 875 three-dimensional thermal-noise image ${ }^{22}$. The intersection of the surface of the pedestal bead 876 with the axis of extension was defined as the anchor position of the tether.

\section{Sources of uncertainty}

878 Our data are subject to several different sources of measurement uncertainty. In the following 879 we refer to the variability within one single-molecule experiments as "precision," whereas we 880 use "accuracy" to refer to the uncertainty between experiments.

The extension of a molecular tether could be measured with the same precision with 882 which the photonic-force microscope could measure the position of the probe bead. To 883 determine this value, we attached a probe bead to a glass coverslip, positioned it in the center 
884 of the position-sensing optical trap, and activated the microscope's drift compensation. Between

$8851 \mathrm{~Hz}$ and $1 \mathrm{MHz}$, the interferometric position signal of the immobilized probe had a band-limited

886 standard deviation of $0.3 \mathrm{~nm}$ along the $\mathrm{y}$-axis, the axis along which we extended single-molecule

887 tethers (Extended Data Table 3a). Over 5 min of observation, the standard deviation for the full

888 bandwidth of DC to $1 \mathrm{MHz}$ was $2 \mathrm{~nm}$ (Extended Data Table 3b).

The accuracy of the position sensor depends on the fidelity of its calibration, which can

890 be tested by its comparison to a calibrated standard. We confined a probe bead in the weak

891 position-sensing optical trap, calibrated the position sensor, and then switched on the high-

892 intensity stimulus trap. This trap was then displaced laterally with respect to the position-sensing

893 trap so that the position sensor reported a displacement of $250 \mathrm{~nm}$. We then compared this

894 nominal displacement to that detected by a camera that acquired brightfield images of the focal

895 plane. The camera had previously been calibrated to accord with well-defined displacements of

896 the nano-positioning stage. Across twelve probe beads, the camera read out an average

897 displacement of $250 \pm 13 \mathrm{~nm}$ (mean \pm SD), values that were in excellent agreement with the

898 microscope's position sensor. We concluded that the bead-to-bead variability of the calibration

899 was $5 \%$ (Extended Data Table 3c). Because the radius of the probe bead was the parameter with

900 the greatest uncertainty during the calibration procedure, this value also set an upper bound of

$9015 \%$ on the coefficient of variation of the diameter of our probe beads.

The pedestal bead scattered a small portion of the position sensing beam, which led to

903 an offset of the position signal that was dependent on the pedestal's position. Although we

904 corrected for this effect through a reference signal, there remained a position offset of $\pm 4 \mathrm{~nm}$

905 peak-to-peak or $3 \mathrm{~nm}$ root-mean-square. This problem contributed uncertainty to the measured

906 displacement between the position-sensing trap and the stimulus trap and thus resulted in

907 reduced accuracy (Extended Data Table 3d). The total accuracy of a nominal distance of $200 \mathrm{~nm}$

908 between the position-sensing trap and stimulus trap was then $10 \mathrm{~nm}$ root-mean-square

909 (Extended Data Table 3e). During a single-molecule experiment, the precision of this distance was 
910 impacted by the slow relative drift between the two optical traps, which we measured as $3 \mathrm{pm} \cdot \mathrm{s}^{-1}$

911 (Extended Data Table 3f).

912 Because the spring constant of the stimulus trap depended linearly on the intensity of the

913 stimulus beam, any variation in the beam's intensity decreased the precision of the spring

914 constant. The spring constant's root-mean-square noise was $0.27 \mu \mathrm{N} \cdot \mathrm{m}^{-1}$ over $20 \mathrm{~s}$ with a

915 vanishingly small drift (Extended Data Table 3g,h). We computed the spring constant from the

916 power-spectral density of the motion of a probe bead as ${ }^{57} k=2 \pi \gamma f_{c}$. The error of the drag $\gamma$

917 was determined by the uncertainty of the probe bead's radius (5\%) and exceeded the error of

918 the corner frequency $f_{c}$. Depending on the radius of the probe bead, the spring constant of the

919 stimulus trap was therefore accurate to within $5 \%$ of the calibrated value (Extended Data

920 Table 3i).

921 We determined the force on the trapped probe bead as the product of its extension from

922 the stimulus trap and the trap's stiffness. Consequently, the precision of the force could be

923 determined from the probe's position noise, the drift of the stimulus trap, and the noise of the

924 trap's spring constant. With the probe bead displaced by $200 \mathrm{~nm}$ from the stimulus trap and for

925 maximal power of the stimulus laser, we determined a precision of $0.7 \mathrm{pN}$ over 5 min of data

926 acquisition (Extended Data Table 3j). During an experiment the displacement of the probe and

927 the power of the laser were usually smaller than those values, resulting in a smaller uncertainty.

928 The accuracy of the force was determined by the total accuracy of the position of the stimulus

929 trap and that of the spring constant. At maximal power of the stimulus laser and for a

930 displacement of the probe bead from the stimulus trap of $200 \mathrm{~nm}$, we found an experiment-to-

931 experiment uncertainty of the force of $3.8 \mathrm{pN}$ (Extended Data Table 3k). For smaller

932 displacements and lower laser powers this uncertainty was lower.

933 Polymer model

934 We modeled PCDH15 as a freely-jointed chain with $N=11$ segments, each of length $b$, 935 representing the eleven stiff cadherin domains ${ }^{34}$. The model included in series a worm-like 936 chain ${ }^{35,59}$ characterized by a persistence length $/ p_{\text {peptide }}$ and a contour length $/ c_{\text {linkers,total }}=10 / c_{\text {linker, }}$, 
which accounted for the ten flexible disordered linker regions between the stiff domains. Any

938 enthalpic extensibility of the protein was described by a Hookean spring with stiffness $k_{\text {folded }}$

939 (Extended Data Fig. 5). Elongation of the protein through the unfolding of each domain was

940 described by the addition of another worm-like chain. Because unfolded polypeptide chains are

941 structurally similar to the inter-domain linker regions, the unfolded portions of the protein were

942 described with an identical persistence length and a contour length of $/ c_{\text {unfolded. }}$

943 The extension-force relation of the protein was described by the sum of the extension-

944 force relations of the freely-jointed chain and of the worm-like chains,

$$
x_{\text {protein }}(F)=x_{\mathrm{FJC}}\left(F, N, b, k_{\text {folded }}\right)+x_{\mathrm{WLC}}\left(F, l p_{\text {peptide }}, l c_{\text {linkers,total }}, k=\infty\right)+
$$

$$
x_{\mathrm{WLC}}\left(F, l p_{\text {peptide }}, l c_{\text {unfolded }}, k=\infty\right) \text {. }
$$

in which the elastic properties of the two worm-like chains were purely entropic $(k=\infty)$. The extension-force relation of an extensible freely-jointed chain is given by ${ }^{34}$

$$
x_{\mathrm{FJC}}(F, N, b, k)=N b\left(\operatorname{coth}\left(\frac{F b}{k_{B} T}\right)-\frac{k_{B} T}{F b}\right)+\frac{F}{k}
$$

950

whereas the elongation of an extensible worm-like chain under force is well approximated by ${ }^{59}$

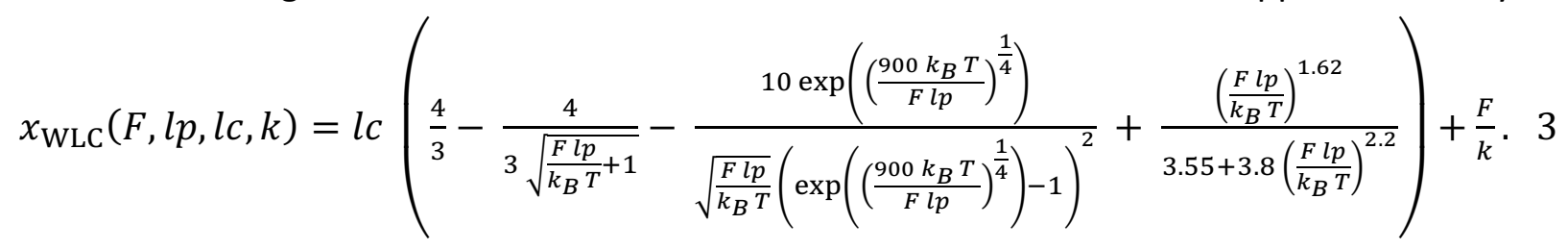

When a force was applied to a single-molecule tether, not only did the tether stretch, but so did the system's remaining elastic elements, the anchors and linkers in series with the tether (Extended Data Fig. 9). We demonstrated experimentally that the combined mechanics of the assay without PCDH15 could be described by an additional extensible worm-like chain (Extended Data Fig. 10), for which we found across nine experiments an average persistence length $/ p_{\text {anchors }}=0.5 \pm 0.1 \mathrm{~nm}$, contour length $/ C_{\text {anchors }}=37 \pm 4 \mathrm{~nm}$, and Hookean spring constant $k_{\text {anchors }}=7.2 \pm 1.3 \mathrm{mNm}^{-1}$ (means $\pm \mathrm{SEMs}$ ).

The total polymer model that we fitted to our data was therefore given by

$$
\begin{gathered}
x_{\text {polymer }}\left(F, N, b, k_{\text {folded }}, l p_{\text {peptide }}, l c_{\text {linkers,total }}, l c_{\text {unfolded }}, l p_{\text {anchors }}, l c_{\text {anchors }}, k_{\text {anchors }}\right) \\
=x_{\text {protein }}\left(F, N, b, k_{\text {folded }}, l p_{\text {peptide }}, l c_{\text {linkers,total }}, l c_{\text {unfolded }}\right)+
\end{gathered}
$$




$$
x_{\mathrm{WLC}}\left(F, l p_{\text {anchors }}, l c_{\text {anchors }}, k_{\text {anchors }}\right) \text {. }
$$

963 Determination of the stiffness of PCDH15 and influence of molecular anchors and beads

964 on the measured stiffness

965 Our force-extension relations capture the mechanics of PCDH15 in series with its molecular 966 anchors and with any compliance of the probe and pedestal beads (Extended Data Fig. 9). To 967 determine the contribution of these elements to our measurements, we conducted experiments 968 without PCDH15 by fusing the amino- and carboxy-terminal anchors and testing their elastic 969 properties in series with the pedestal and probe beads (Extended Data Fig. 10). PCDH15's 970 stiffness could then be determined by treating the whole system as a series of nonlinear springs,

$$
k_{P C D H 15}(F)=\frac{k_{\text {total }}(F) \cdot k_{\text {anchors }}(F)}{k_{\text {anchors }}(F)-k_{\text {total }}(F)} .
$$

973 For $\mathrm{Ca}^{2+}$ concentrations of $3 \mathrm{mM}$ and $20 \mu \mathrm{M}$, we computed the stiffness of the full-length 974 construct - the protein in series with its anchors and the beads-as the spatial derivative of the 975 mean of the force-extension relations associated with its different conformational states 976 (Extended Data Fig. 11). Computed in an analogous way (Extended Data Fig. 10d), the stiffness of 977 the construct without PCDH15 was much larger (Extended Data Fig. 11c,d). From the values of 978 State 1 we computed the stiffness of folded PCDH15 and found that it is surprisingly soft under 979 physiological tensions, offering a stiffness between $0.5 \mathrm{mN} \cdot \mathrm{m}^{-1}$ at a tension of $5 \mathrm{pN}$ and $6 \mathrm{mN} \cdot \mathrm{m}^{-1}$ 980 at $50 \mathrm{pN}$ (Figure 3a,b).

982 The position and force signals (Extended Data Fig 1b,c), sampled at $100 \mathrm{kHz}$, were split into 983 individual extension-relaxation cycles and smoothed with a second order Savitzky-Golay filter 984 over a window of 101 points, which reduced the temporal resolution to 1 ms. Conformational 985 changes of the protein were automatically detected as sudden increases or decreases in the 986 filtered probe-position signal. To determine whether a structural change occurred at point $i$ of 
the time trace, we computed the averages and standard deviations of 1000 points preceding and

988 succeeding $i,\langle x\rangle_{\text {left }},\langle x\rangle_{\text {right }}, \sigma_{\text {left, }}$ and $\sigma_{\text {right. }}$ A structural change was detected at point $i$ if

$$
\left|\langle x\rangle_{\text {left }}-\langle x\rangle_{\text {right }}\right|>4 \frac{\sigma_{\text {left }}+\sigma_{\text {right }}}{2} \text {. }
$$

990 Structural changes that did not fulfill this criterion were missed by our algorithm. If two or more

991 structural changes occurred within 10 ms of one another, our algorithm detected only one larger 992 event.

We then segmented each extension-relaxation cycle into its individual states, demarcated

994 by the structural changes, and fit our polymer model to each of the segments. To facilitate meaningful fits, we sought to constrain the number of free parameters in our polymer model

996 (Equation 4) as much as possible. We first independently measured the parameter values of the 997 worm-like chain describing the compliance of the anchors and of the rest of the assay without 998 PCDH15 (Extended Data Figs. 9 and 10), for which we found an average persistence length $999 / p_{\text {anchors }}=0.5 \pm 0.1 \mathrm{~nm}$, contour length $/ C_{\text {anchors }}=37 \pm 4 \mathrm{~nm}$, and Hookean spring constant $1000 \quad k_{\text {anchors }}=7.2 \pm 1.3 \mathrm{mNm}^{-1}$ (means \pm SEMs of nine experiments). We held these values constant at 1001 their means for all subsequent fits of extension-relaxation cycles of PCDH15. In a next step it was important to determine / $p_{\text {peptide, }}$ the persistence length of PCDH15's 1003 unfolded polypeptide chains, for this value entangles the mechanics of State 1 (through the linker 1004 regions of the folded protein) with the mechanics of partially unfolded PCDH15. To facilitate this 1005 determination, we temporarily approximated State 1 of the protein as a worm-like chain; we 1006 then held the parameter values of State 1's worm-like chain model constant while fitting all 1007 extension-relaxation cycles with only $I p_{\text {peptide }}$ and $/ C_{\text {unfolded }}$ as free parameters, determining $1008 I p_{\text {peptide }}=0.49 \pm 0.04$ (mean $\left.\pm \mathrm{SEM}, N=13\right)$. Because there was no apparent difference in peptide 1009 persistence length between the results for $\mathrm{Ca}^{2+}$ concentrations of $3 \mathrm{mM}$ and $20 \mu \mathrm{M}$, data were 1010 averaged across 13 experiments at both concentrations. This value of $I p_{\text {peptide }}$ was held constant 1011 at its mean for all subsequent fits.

1012 We then fitted State 1 with our protein model to determine the remaining free 1013 parameters of the folded protein, $I_{\text {linker }}, b$, and $k_{\text {folded }}$ (Table 1$)$. Finally, we fitted all segments of 
1014 all extension-relaxation cycles with the polymer model, with $/ c_{\text {linker }}, b$, and $k_{\text {folded }}$ held constant at

1015 the values determined for the individual proteins, with $/ c_{\text {unfolded }}$ as the only free parameter. The

1016 changes of $/ c_{\text {unfolded }}$ between adjacent segments then described the end-to-end elongation of the

1017 tether due to unfolding events (Fig. 2).

1018 Statistical dependence of events $A_{U}$ and $B_{U}$

1019 In order to test whether the order of occurrence of events $A_{\cup}$ and $B_{\cup}$ is statistically significant, 1020 we simulated extension trials with independent probability densities for the two events such that 1021 the resulting force distributions for each of the classes of rips was identical to our experiments. 1022 We then showed that the sequence of events in these simulations did not match our 1023 experimental observations, proving that the probability densities underlying our experiments are 1024 statistically dependent.

For every simulated trial, we first drew two random numbers to determine whether both 1026 rips would occur during the extension. The probabilities $p_{A}$ and $p_{B}$ for respective events $A_{U}$ and 1027 Bu were determined separately from the experimental data for each PCDH15 molecule. For 1028 traces that contained both rips, a parameter representing the pulling force was then linearly 1029 increased from $0 \mathrm{pN}$ to $80 \mathrm{pN}$ in steps $d F=0.1 \mathrm{pN}$. After each force increment we allowed rips $\mathrm{A}_{U}$ 1030 and $\mathrm{B} \cup$ to occur with independent probabilities $\rho_{A}(F) d F$ and $\rho_{B}(F) d F$, in which $\rho_{\mathrm{A}, \mathrm{B}}$ represent 1031 probability densities. Because each class of rips represents a unique structural change, once a rip 1032 of either type had occurred no further event of that class could follow. The simulated record was 1033 discarded if neither or only one of the rips occurred over the simulated force range. The 1034 probability densities $\rho_{A, B}(F)$ were independently determined for each protein and could be 1035 calculated from the experimentally observed force histograms of rips $N_{A, B}(F)$ :

1036

$$
N_{A, B}(F)=\rho_{A, B}(F) d F \cdot\left(1-\int_{0}^{F} \rho_{A, B}\left(F^{\prime}\right) d F^{\prime}\right) \cdot N \cdot p_{A, B}
$$

1037 in which $\rho_{A, B}(F) d F$ is the probability of finding rip $A_{\cup}\left(\right.$ or $B_{\cup}$ ) at force $F$ and $N \cdot p_{A, B}$ is the number 1038 of extension trials that contain rip $A_{\cup}$ (or $B_{\cup}$ ). The factor in parentheses is the probability that at 
1039 force $F$ during an extension a rip has not yet occurred at forces lower than $F$. This factor accounts

1040 for the fact that we draw from $\rho_{A, B}(F)$ for linearly increasing $F$, and stop drawing from the

1041 distribution when a rip occurs. We accordingly can draw from $\rho_{A, B}(F)$ only if no other rip

1042 occurred at a lower force. It follows that

$$
\rho_{A, B}(F)=\rho_{0 A, B}(F)+\rho_{A, B}(F) \int_{0}^{F} \rho_{A, B}\left(F^{\prime}\right) d F^{\prime}
$$

1044 in which $\rho_{0 A, B}(F)=\frac{N_{A, B}(F)}{N \cdot p_{A, B} \cdot d F}$ are the normalized force histograms of the experimentally observed 1045 rips. We numerically solved this relation to determine $\rho_{A, B}(F)$ (Extended Data Fig. 12a,c).

Our simulations successfully reproduced the experimentally observed force histograms

1047 of the rips $A_{\cup}$ and $B_{\cup}$ (Extended Data Fig. 12b,d). We then enumerated the trials in which rip $A_{U}$

1048 occurred before rip Bu. For the molecule shown in the left column of Figure 2, our simulations-

1049 based on the independent probability distributions $\rho_{A}(F)$ and $\rho_{B}(F)$-predicted that we should

1050 have observed 20.4 extensions in which $A_{\cup}$ preceded $B_{\cup}$, a contradiction to the observed value

1051 of $8(p<0.05)$. Because the counts were assumed to be Poisson distributed, we made statistical

1052 comparisons by computing the Wald test statistic $Z=\frac{N_{o}-N_{p}}{\sqrt{N_{o}+N_{p}}}$, in which $N_{o}$ are the observed

1053 counts and $N_{p}$ are the predicted counts, followed by a two-tailed comparison of $Z$ to a normal

1054 distribution to compute the $p$-value. We therefore reject the assumption that $A_{\cup}$ and $B_{\cup}$ are

1055 independent. An identical conclusion was reached for all five proteins tested at a $\mathrm{Ca}^{2+}$

1056 concentration of $3 \mathrm{mM}$, although two were significant only at $p<0.1$. When we tested proteins

1057 at $20 \mu \mathrm{M}\left[\mathrm{Ca}^{2+}\right]$ in the same manner, we again routinely observed fewer trials in which rip $A_{U}$

1058 occurred before rip $\mathrm{B}_{\cup}$ than predicted by our simulations. At this physiological $\mathrm{Ca}^{2+}$ concentration,

1059 however, the difference between the observed and expected number of sequences $A B$ was no

1060 longer statistically significant at $p<0.1$.

1061 Transformation of force histograms into force-dependent rate constants

1062 We transformed the measured distribution of rip forces of type $C_{U}$ into force-dependent 1063 unfolding rate constants ${ }^{28}$. PCDH15 consists of 11 domains that we approximated as equivalent 1064 for this analysis. In this approximation, they are each assumed to be equally likely to unfold under 
1065 force. Hence, a fully folded PCDH15 molecule is eleven times more likely to unfold a domain than

1066 a PCDH15 molecule with only one folded domain. To arrive at correct values for the unfolding

1067 rates of individual cadherin domains, we therefore computed individual force histograms of $C_{U}$

1068 events originating from each of the states of the protein. We then weighted the measured force

1069 histograms with the number of folded cadherin domains in each state: for example, the force

1070 histogram for domain unfolding from States 1 and 2, which both correspond to PCDH15 with

1071 eleven (at least partially) folded cadherin domains, was divided by $n=11$ domains. The weighted

1072 force histograms were then combined and the remaining analysis performed ${ }^{28}$. Our weighting of

1073 the force histograms is similar to the weighting that has been employed in pseudo dwell time

1074 analysis for the determination of un- and refolding rates in chains of identical titin domains ${ }^{33}$. 


\section{Extended Data}

\section{Extended Data figures and legends}
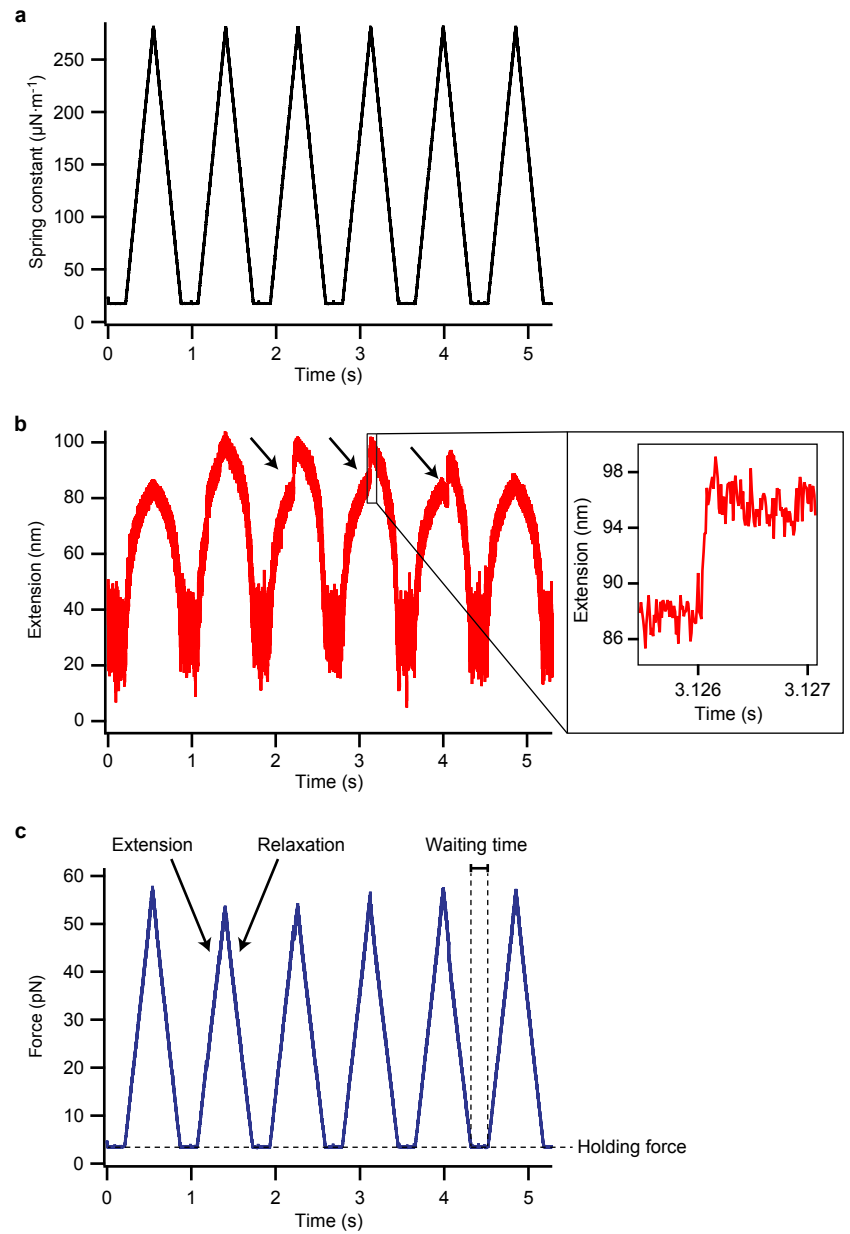

Extended Data Fig. 1 | Time traces of extension-relaxation cycles. (a) The force on a PCDH15

1080 tether was varied by adjustment of the spring constant of the stimulus trap, which was centered

1081 about two hundred nanometers from the equilibrium position of the probe bead. (b) The tether's

1082 extension included occasional abrupt events (arrows). Note the extensive noise owing to thermal

1083 excitation of the molecule. (c) The force acting on the tethered protein was computed from the

1084 probe bead's position and the spring constant of the stimulus trap. Each protein underwent

1085 hundreds of extension-relaxation cycles, between which the force was held constant for a 1086 particular waiting time so that any unfolded domains could refold. The $\mathrm{Ca}^{2+}$ concentration was $10873 \mathrm{mM}$. 


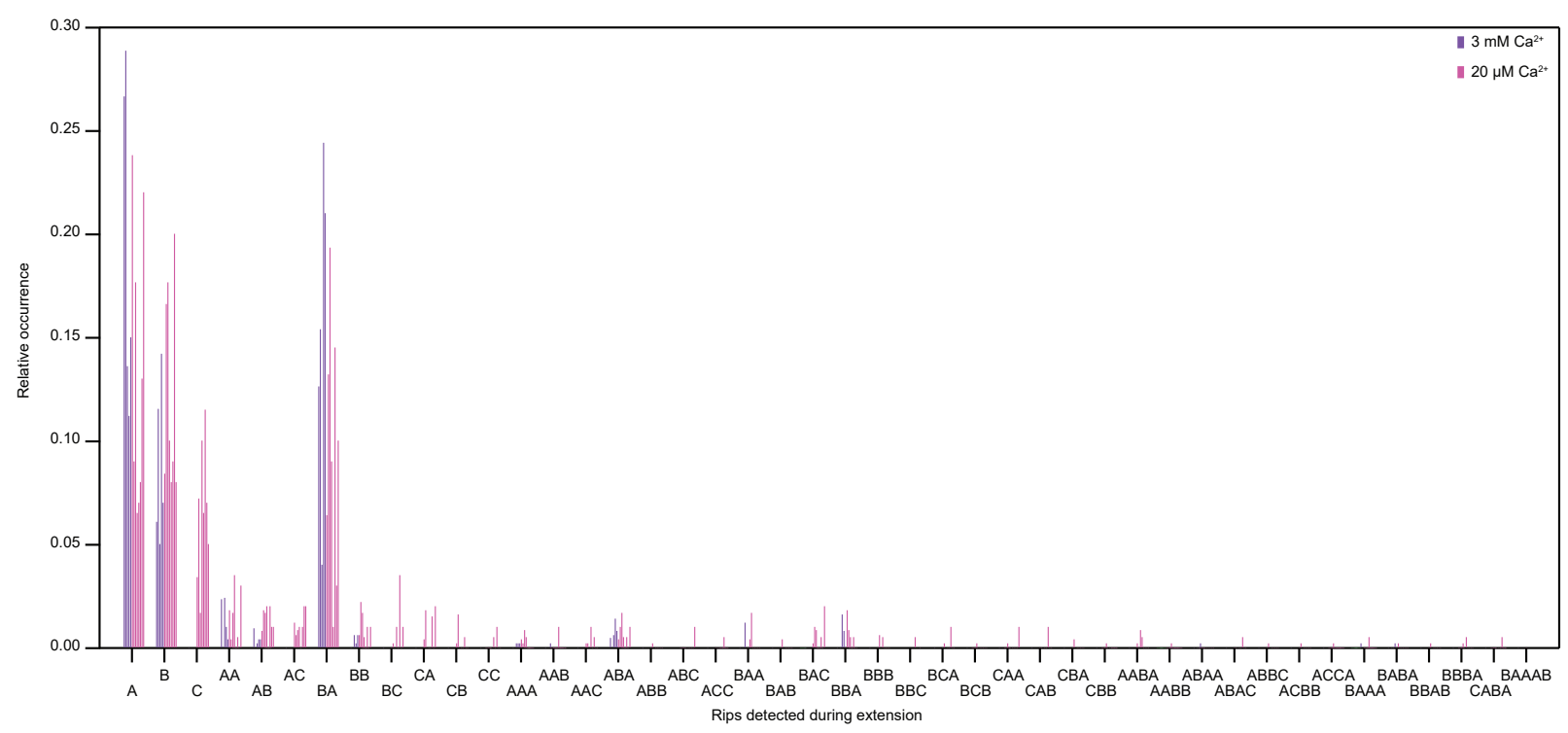

1089 Extended Data Fig. 2 | Sequences of structural changes during extension trials. The histogram

1090 indicates the fraction of extension traces that contained a specific sequence of unfolding events.

1091 For each possible sequence of events, each bar in a cluster represents a single molecule. Each

1092 molecule was extended at a loading rate of $130 \mathrm{pN} \cdot \mathrm{s}^{-1}$. Depending on the protein, the holding

1093 force between cycles was 2-4 pN and the waiting time was 0.2-2 s. This inconsistency altered the

1094 extent to which a particular molecule refolded between cycles and added variability to the 1095 results. 


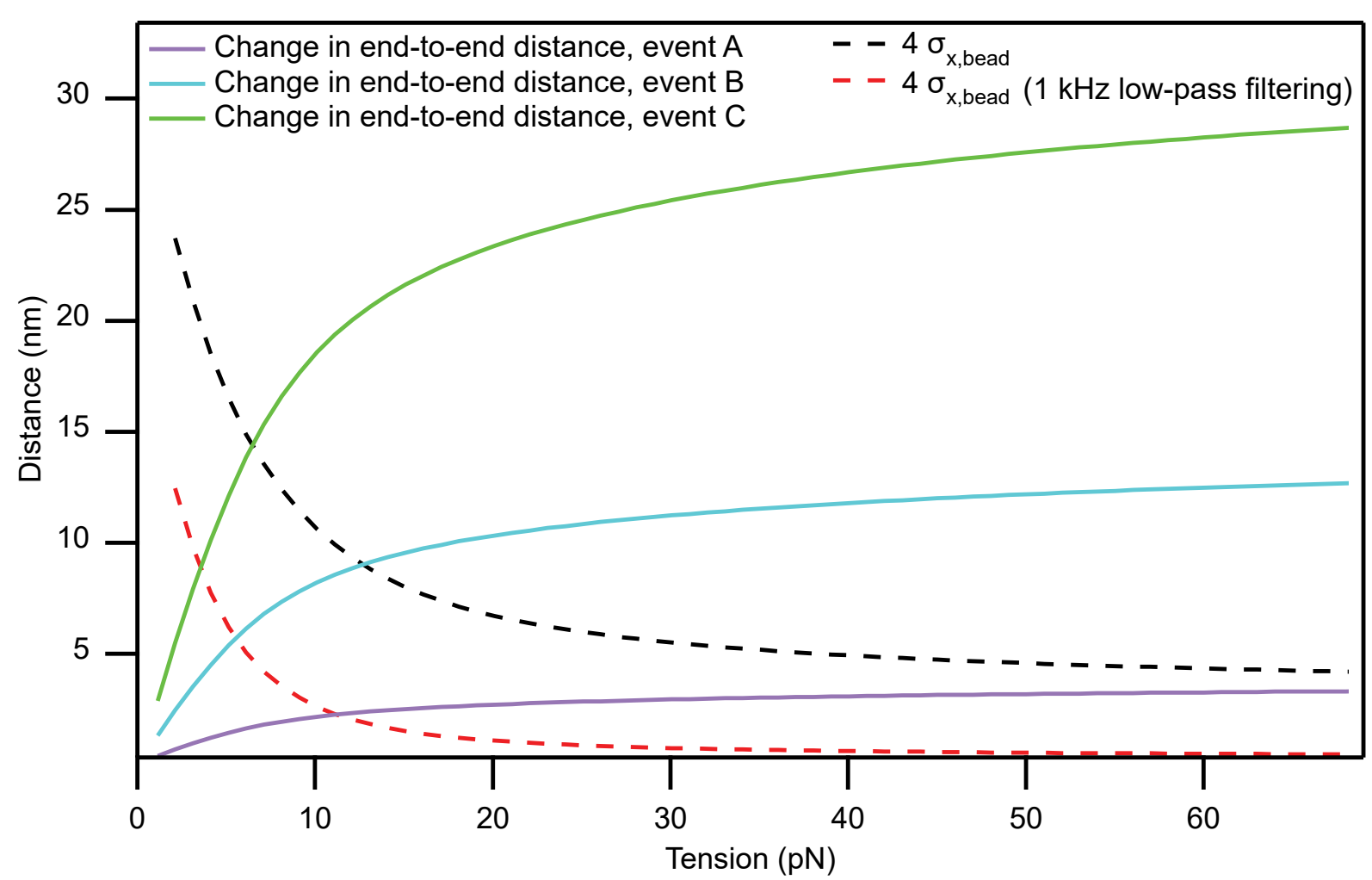

1098 Extended Data Fig. 3 | Effect of thermal noise on detectability of conformational changes.

1099 When a molecular tether is subjected to a low tension, unfolding or refolding of a domain results

1100 in a change in the end-to-end distance much smaller than the actual change in contour length.

1101 The expected changes (solid lines) are shown for events of types A, B, and C. If these changes are

1102 comparable to the thermal motion of the bead, they cannot be reliably detected by our method.

1103 We can confidently identify a folding event if the change in end-to-end distance is larger than

1104 four times the standard deviation of the thermal motion (black dashed line, $1 \mathrm{MHz}$ bandwidth;

1105 dashed red line, signal low-pass filtered to $1 \mathrm{kHz}$ ). The intersection of the dashed and solid lines

1106 therefore defines the force below which a given structural change can no longer be reliably

1107 resolved for a particular temporal resolution. The band-limited thermal noise in the probe bead's

1108 position was computed as the integral of the power-spectral density of the bead's motion ${ }^{57,53}$. 


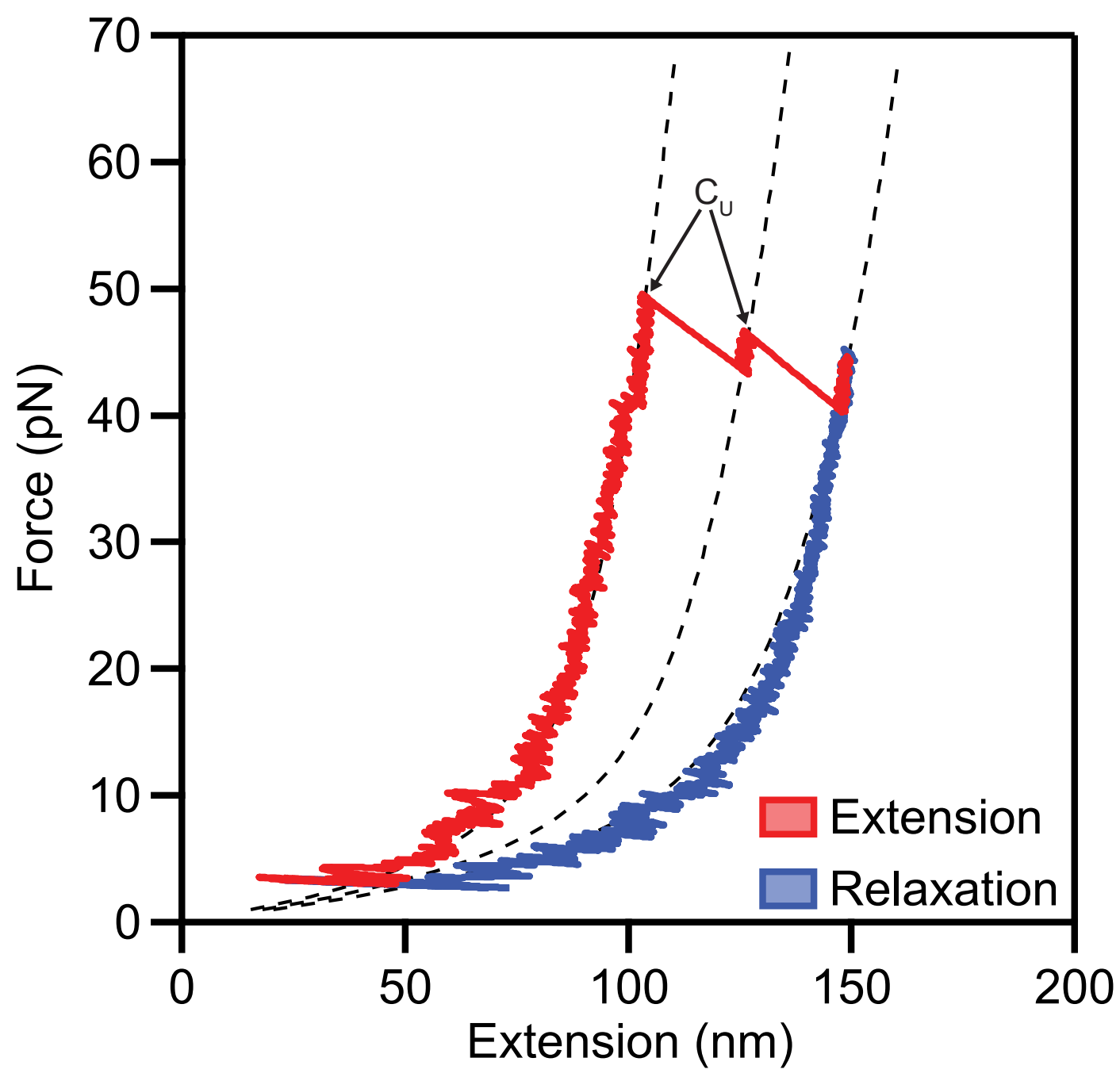

1111 Extended Data Fig. 4 | Unfolding of several cadherin domains during one extension-relaxation

1112 cycle. In some extension-relaxation cycles of the protein from Fig. 2b, two cadherin domains

1113 unfolded during the extension (arrows). The $\mathrm{Ca}^{2+}$ concentration was $20 \mu \mathrm{M}$. 
a

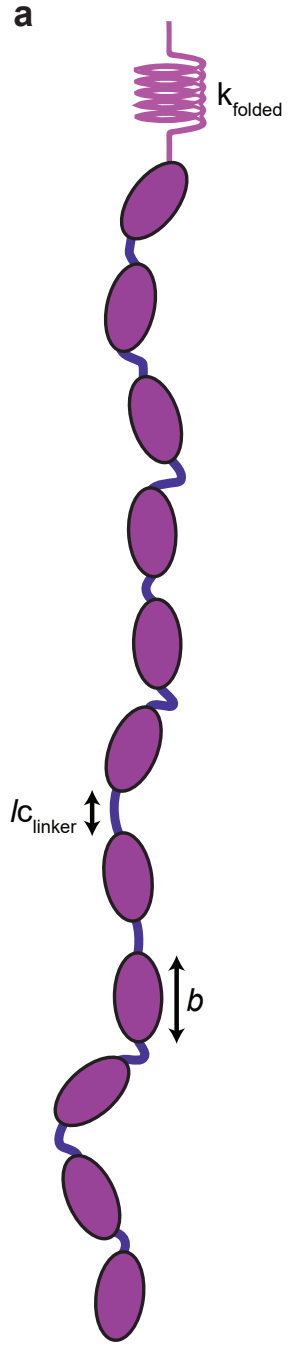

b

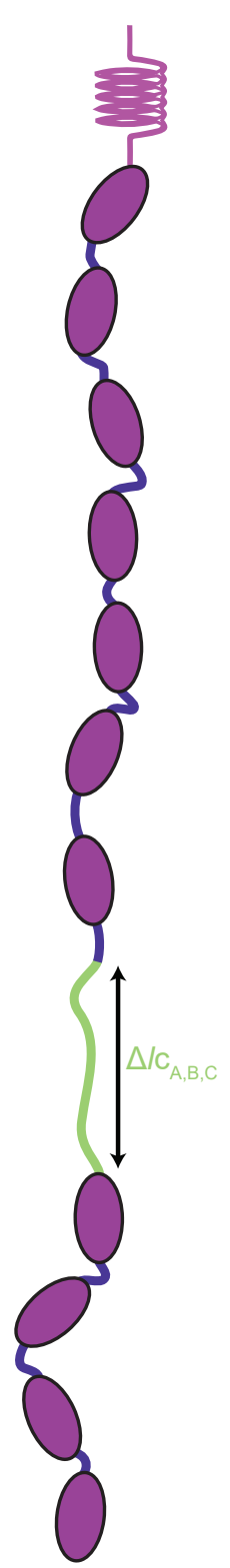

1116 Extended Data Fig. 5 | Protein model for PCDH15 under tension. (a) We modeled a folded

1117 monomer as a freely jointed chain of eleven stiff segments, each with length $b$. The linker regions

1118 between stiff segments consist of unstructured peptides of length $/ c_{\text {linker, }}$ whose combined effect

1119 was modeled as a worm-like chain with a contour length of $10 \cdot / c_{\text {linker }}$ and persistence length

$1120 \mathrm{Ip}=0.49 \mathrm{~nm}$ in series with the freely-jointed chain. A Hookean spring with stiffness $k_{\text {folded }}$

1121 represents the enthalpic extensibility of the protein. (b) Each unfolding event was represented

1122 as an additional worm-like chain (green), of the appropriate contour length and of persistence

1123 length $0.49 \mathrm{~nm}$, in series with the folded protein. 


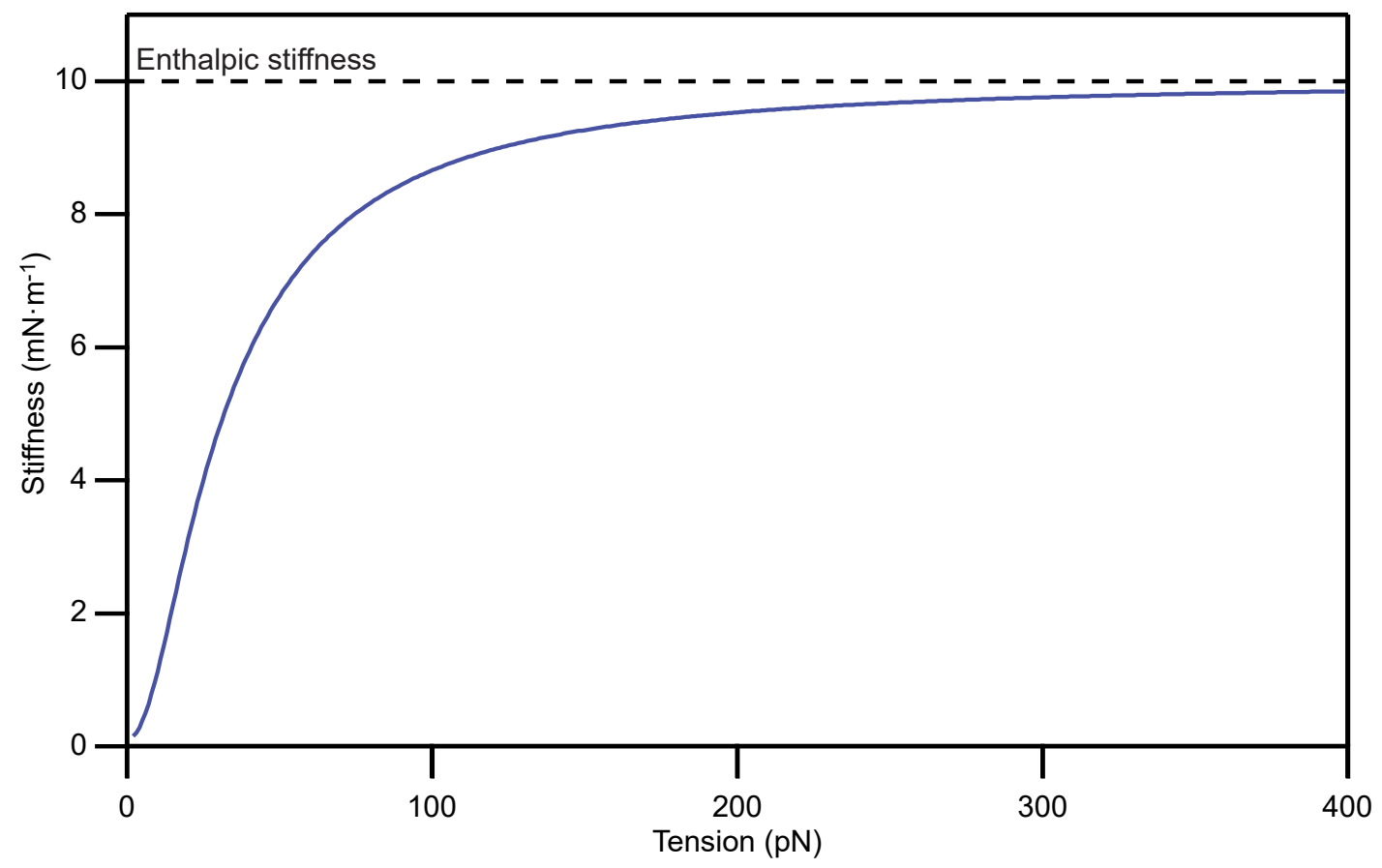

1125 Extended Data Fig. 6 | Predicted stiffness of PCDH15 at unphysiologically high forces. Our

1126 model predicts that monomeric PCDH15 reaches its enthalpic stiffness only for tensions 1127 exceeding hundreds of piconewtons. The physiological range of tensions, $4 \mathrm{pN}$ to $25 \mathrm{pN}$ per 1128 molecule, is dominated by the protein's entropic elasticity. 


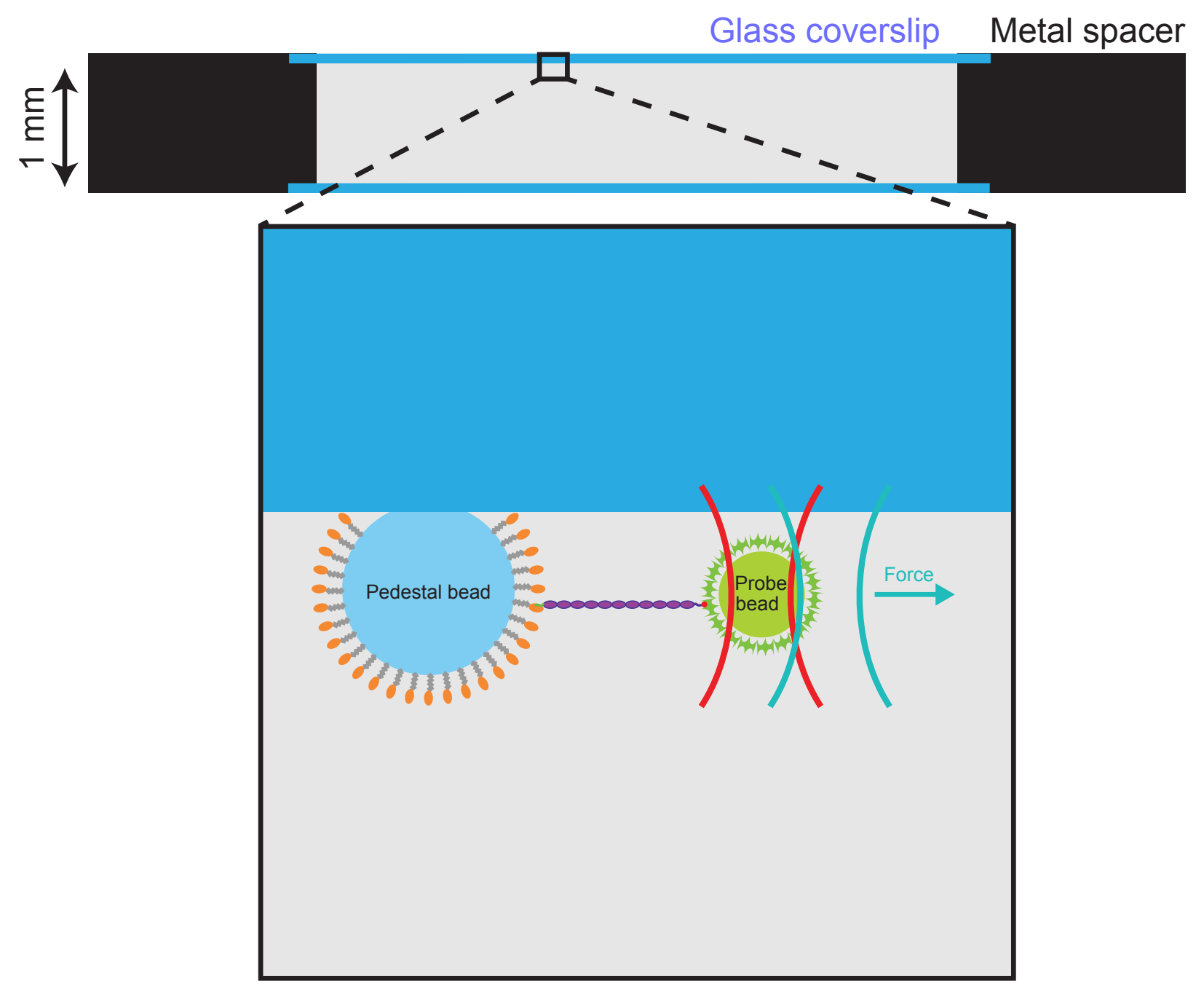

1131 Extended Data Fig. 7 | Design of the sample chamber. The sample chamber consisted of two

1132 glass coverslips attached by vacuum grease to a metal spacer. The sparsely distributed pedestal

1133 beads were covalently attached to the functional surface of the upper coverslip, and the chamber

1134 was filled with buffer solution containing freely diffusing probe beads. Note that the photonic-

1135 force microscope was of upright design, with the objective lens positioned above the sample

1136 chamber. 
a

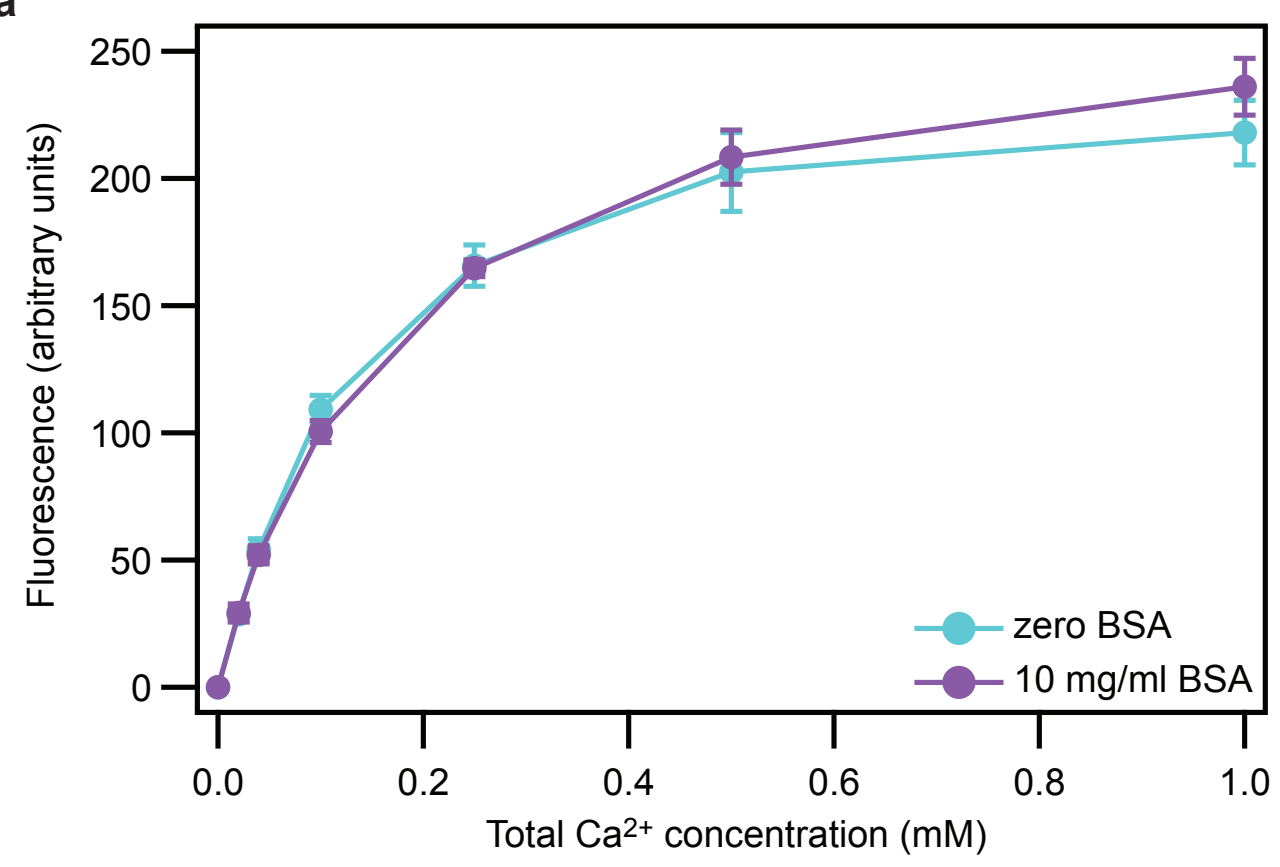

b

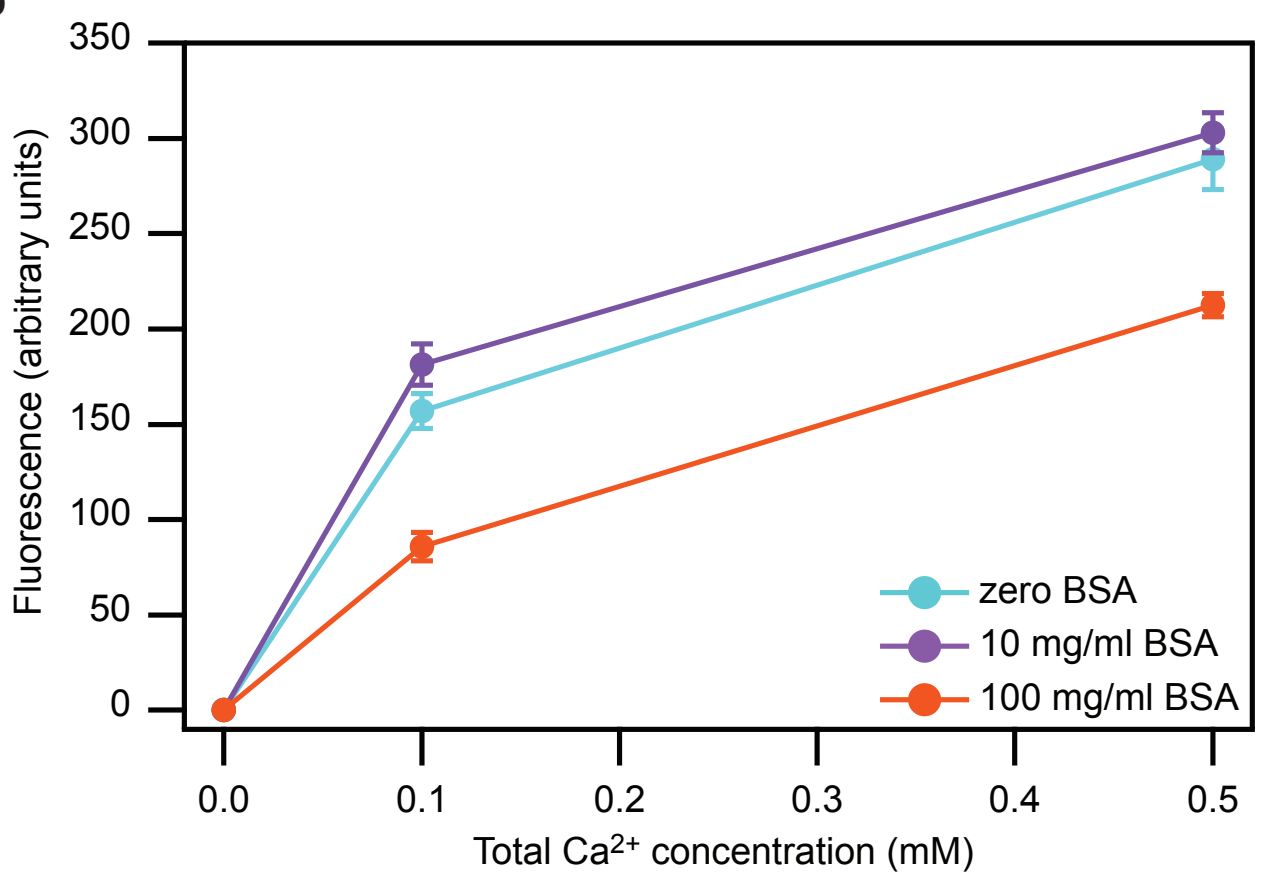

1139 Extended Data Fig. 8 | Lack of effect of bovine serum albumin (BSA) on the concentration of

1140 free $\mathrm{Ca}^{2+}$ ions. (a) The fluorescence of the $\mathrm{Ca}^{2+}$ indicator Fluo-5N (F14203, ThermoFisher

1141 Scientific, Waltham, MA, USA) is unchanged in the presence of $10 \mathrm{mg} / \mathrm{ml}$ BSA. (b) The

1142 concentration of free $\mathrm{Ca}^{2+}$ is diminished only by the presence of a BSA concentration as great as

$1143100 \mathrm{mg} / \mathrm{ml}$. Data are plotted as means \pm SDs for three experiments. 
a

Extended Data Fig. 9 | Compliant elements in the single-molecule assay. (a) To test the stiffness of PCDH15, we confined an individual monomer between a pedestal and a probe bead. Because each element of the single-molecule assay is compliant, however, the stiffness of the rest of the

1148 system - without the PCDH15-must be known in order to accurately determine the protein's

1149 stiffness. The components are not drawn to scale. (b) The assay's compliant elements include the 1150 covalent anchoring of the glass pedestal bead to the coverslip, the linkage between the pedestal 1151 bead and carboxy-terminus of the protein (polyethylene glycol [PEG], SpyCatcher, SpyTag, and 1152 peptide), and the connection between the protein's amino-terminus and the probe bead 1153 (peptide, biotin, and streptavidin). The latter linkage might also include a short polystyrene tether 1154 that extends from the probe bead's surface. (c) We designed the assay to contain anchor and 1155 linker elements that were as short and stiff as possible. Their approximate contour lengths are 1156 indicated. The size of the SpyCatcher, PCDH15, and streptavidin proteins were estimated from 1157 their crystal structures (PDB IDs 4MLI, 6CV7, and 1AVD). The lengths of the PEG and peptides are 1158 design lengths, and the length of the polystyrene tether was estimated from control experiments 1159 (Extended Data Fig. 10). 
a

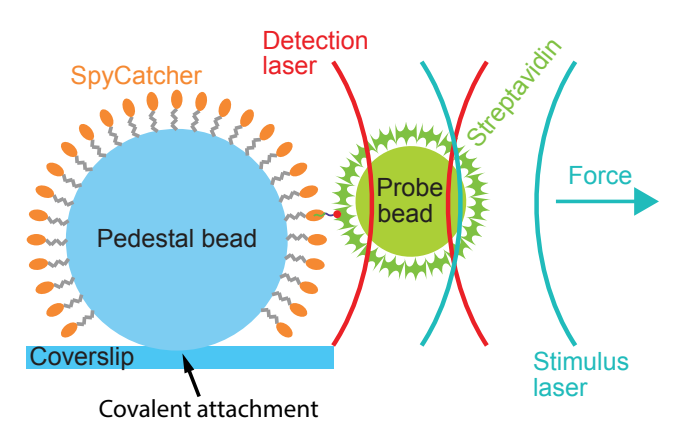

C

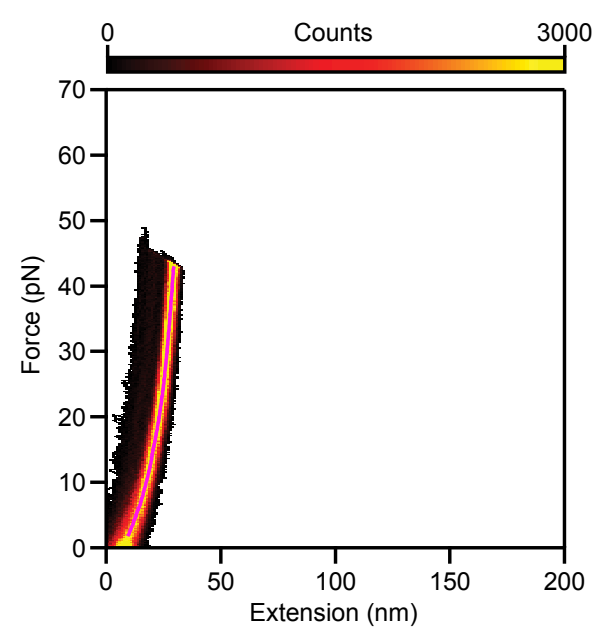

b

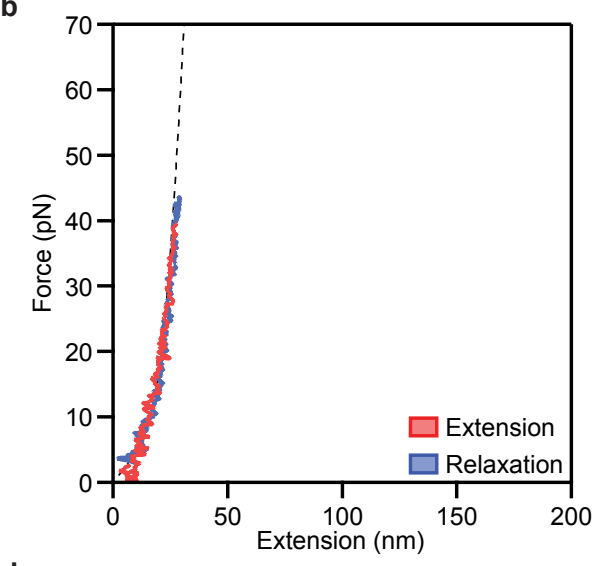

d

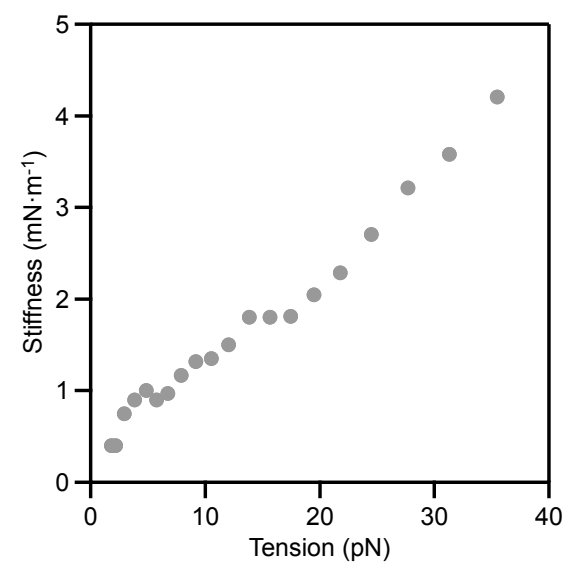

1161 Extended Data Fig. 10 | Mechanical properties of the anchors and linkers. (a) In order to

1162 measure the stiffness of the single-molecule assay system in the absence of PCDH15, we

1163 connected the molecular handles (SpyTag and biotinylation peptide) through an eight-amino-

1164 acid, flexible linker, anchored this short peptide between a pedestal and a probe bead, and

1165 determined its force-extension relation. (b) A representative extension-relaxation cycles features

1166 a small amount of extensibility that is well fit by a wormlike-chain model. Across nine

1167 experiments we found an average persistence length $/ p_{\text {anchors }}=0.5 \pm 0.1 \mathrm{~nm}$, contour length

$1168 / C_{\text {anchors }}=37 \pm 4 \mathrm{~nm}$, and Hookean spring constant $k_{\text {anchors }}=7.2 \pm 1.3 \mathrm{mNm}^{-1}$ (means $\pm S E M s$ ). The

1169 designed contour length of our tether including PEG, SpyCatcher, peptide, and streptavidin is

$117020 \mathrm{~nm}$, shorter than the tether's experimentally determined contour length of $37 \mathrm{~nm}$. We

1171 accordingly conclude that the extensible polystyrene hairs on the surface of the probe beads

1172 have an average length of $17 \mathrm{~nm}$ (Extended Data Fig. 9). (c) A state-space heatmap of 500 
1173 extension-relaxation cycles shows only the single conformational state expected for an

1174 unstructured peptide. These data confirm that neither of our proteinaceous anchors undergoes

1175 structural changes over the relevant force range. (d) To estimate the compliance of our system

1176 of anchors, we determined the average of the highly occupied region in the heat map (pink line

1177 in c) and computed its slope.

1178 
a

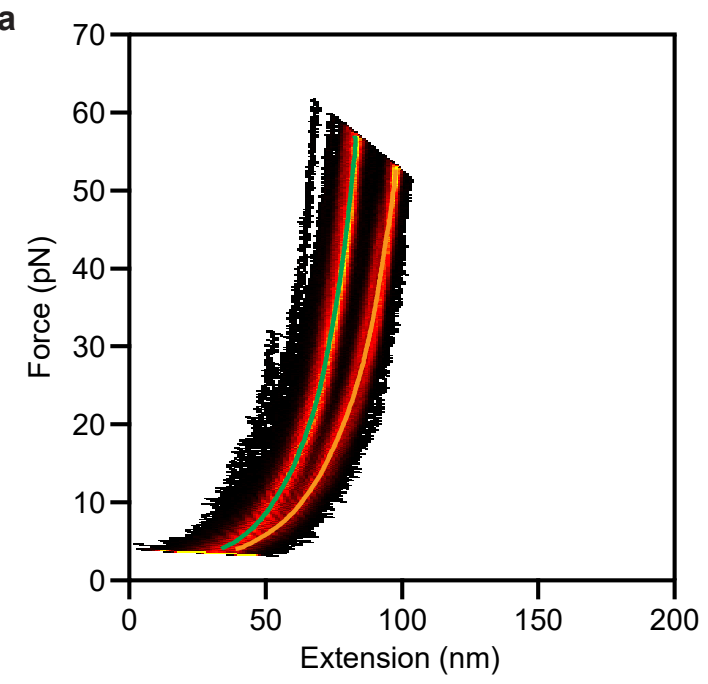

b

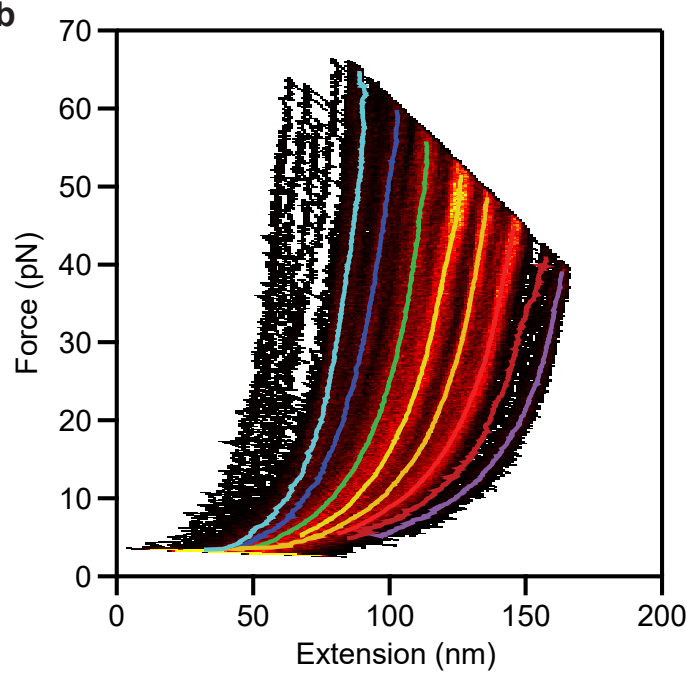

C

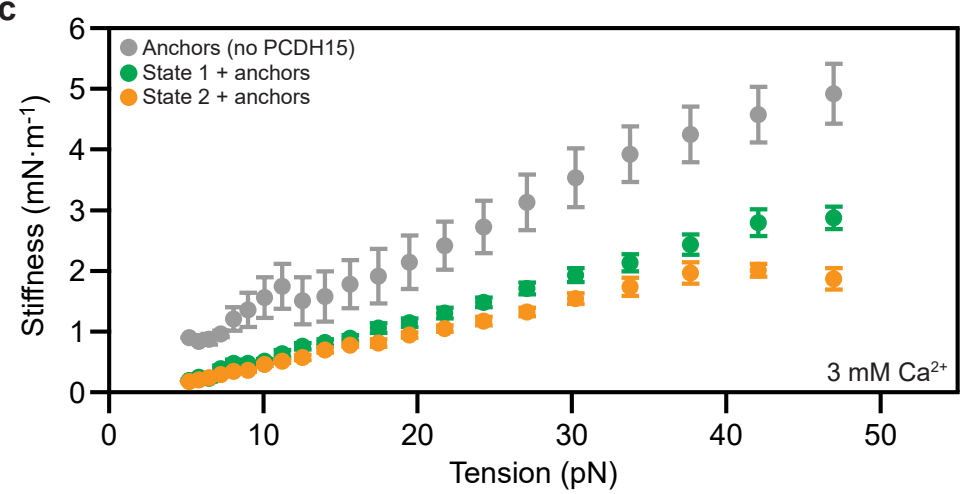

d

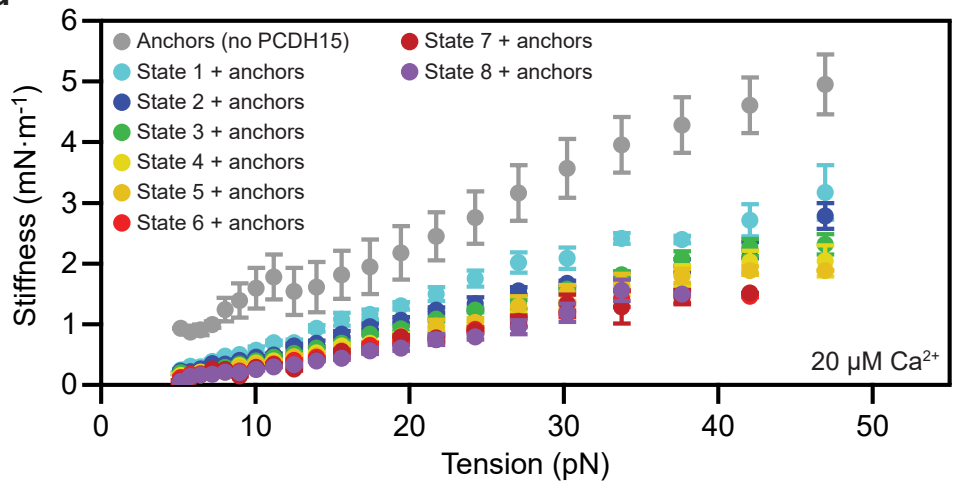

1180 Extended Data Fig. 11 | Total stiffness of the single-molecule assay system. (a) We determined

1181 the stiffness of PCDH15 in its different conformational states at a $\mathrm{Ca}^{2+}$ concentration of $3 \mathrm{mM}$ by

1182 finding the slope of each highly occupied region of the state-space heatmap. (b) The same

1183 operation was performed for $\mathrm{PCDH} 15$ in the presence of $20 \mu \mathrm{M} \mathrm{Ca}^{2+}$. (c) The resulting total

1184 stiffnesses represent the compliances of the anchors and of PCDH15 at a $\mathrm{Ca}^{2+}$ concentration of

$11853 \mathrm{mM}$. (d) The result for $20 \mu \mathrm{M}\left[\mathrm{Ca}^{2+}\right]$ includes six additional unfolded states of progressively 
1186 diminishing stiffness. As expected, for all states the total stiffness is lower than the stiffness of

1187 the anchors alone. By treating PCDH15 and its anchors as springs in series, we can compute from

1188 these data the stiffness of PCDH15 alone. The data in $\mathbf{c}$ and $\mathbf{d}$ are means \pm SEMs for five molecules

1189 and six molecules at a $\mathrm{Ca}^{2+}$ concentration of respectively $3 \mathrm{mM}$ and $20 \mu \mathrm{M}$.

1190 
a

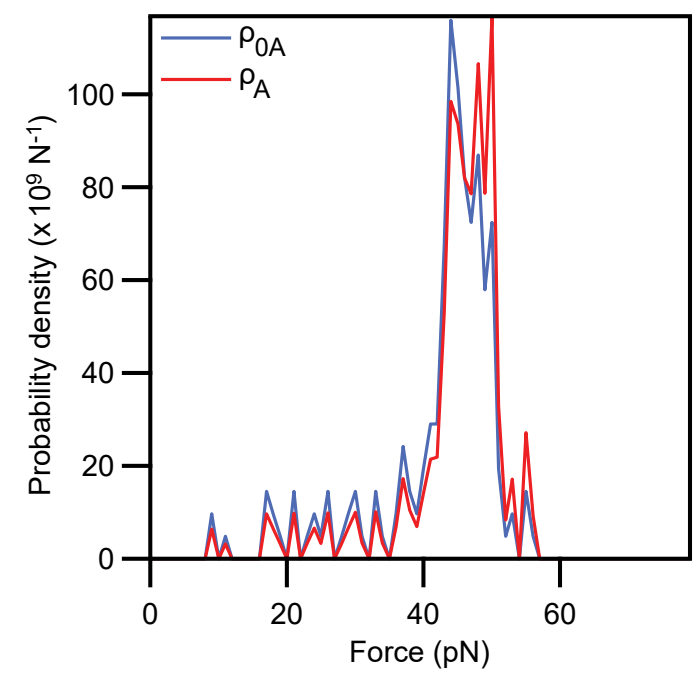

C

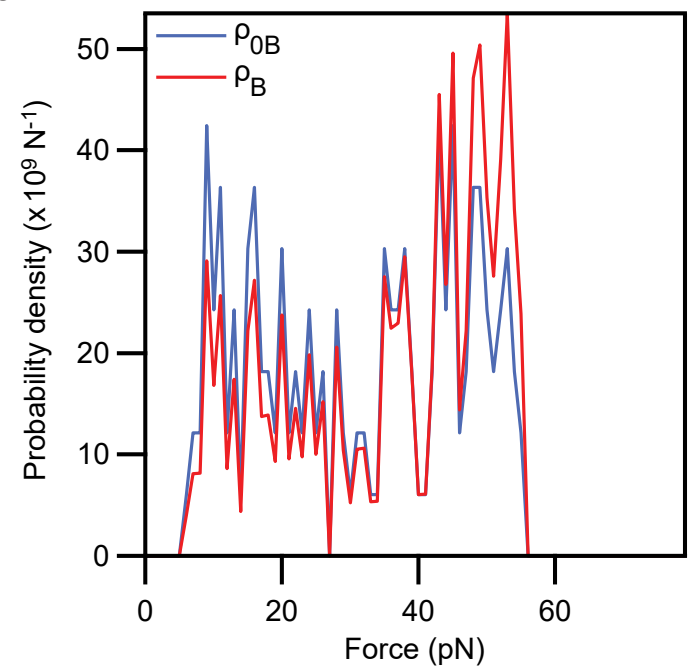

b

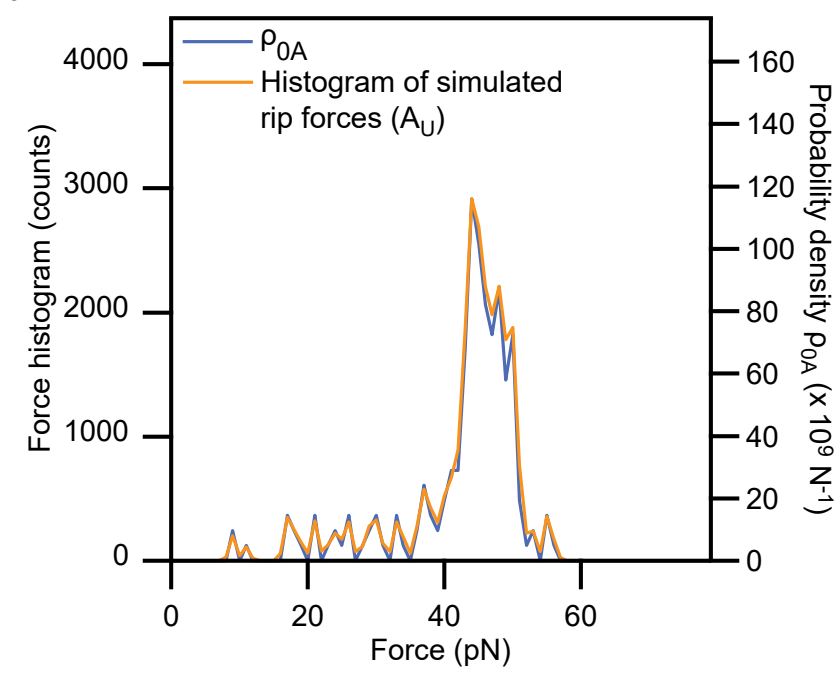

d

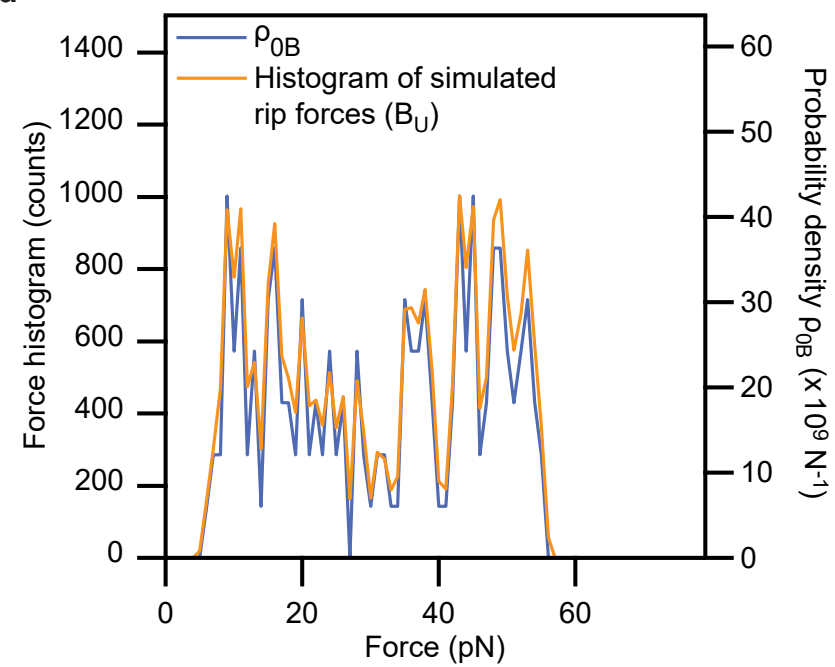

1192 Extended Data Fig. 12 | Simulation of the force distributions of the unfolding events $A_{u}$ and $B_{u}$.

1193 (a) During an extension, a rip of class A occurs with probability $\rho_{A}(F) d F$. We calculated the 1194 probability density $\rho_{A}(F)$ from the experimentally observed force histogram of rips, $\rho_{0 A}(F)$.

1195 (b) To confirm that our simulations successfully reproduced the experimentally observed force 1196 histograms, we simulated 200,000 extension trials, in each of which rips occurred with a 1197 probability $\rho_{A}(F) d F$. (c) A similar procedure was applied to events of class B. (d) A histogram 1198 displays the distribution expected from the simulation. For both classes of unfolding event, the 1199 histograms of simulated rip forces match the experimentally observed force histograms. 
1201 Extended Data Tables

1202 Extended Data Table 1 | Confidence of tethering a single PCDH15 molecule rather than 1203 several ${ }^{58}$.

\begin{tabular}{|l|l|l|}
\hline $\mathrm{Ca}^{2+}$ concentration & Sample number & Confidence of single-molecule tethers \\
\hline 0 & 1 & $>90 \%$ \\
\hline 0 & 2 & $>85 \%$ \\
\hline 0 & 3 & $>95 \%$ \\
\hline 0 & 4 & $>70 \%$ \\
\hline 0 & 5 & $>85 \%$ \\
\hline $20 \mu \mathrm{M}$ & 6 & $>85 \%$ \\
\hline $20 \mu \mathrm{M}$ & 7 & $>85 \%$ \\
\hline $20 \mu \mathrm{M}$ & 8 & $>95 \%$ \\
\hline $20 \mu \mathrm{M}$ & 9 & $>90 \%$ \\
\hline $20 \mu \mathrm{M}$ & 10 & $>95 \%$ \\
\hline $3 \mathrm{mM}$ & 11 & $>95 \%$ \\
\hline $3 \mathrm{mM}$ & 12 & $>85 \%$ \\
\hline $3 \mathrm{mM}$ & 13 & $>90 \%$ \\
\hline $3 \mathrm{mM}$ & 14 & $>95 \%$ \\
\hline
\end{tabular}


1206 Extended Data Table 2 | Confinement of PCDH15 molecules through biotin-streptavidin

1207 and SpyCatcher-SpyTag interactions. We tested whether PCDH15 was specifically

1208 confined between pedestals and probe beads through its amino- and carboxy-terminal

1209 tags. Tether formation was abolished if components of either the SpyCatcher-SpyTag or

1210 of the biotin-streptavidin pair were missing.

1211

\begin{tabular}{|c|c|c|c|c|c|c|}
\hline & \multicolumn{2}{|c|}{$\begin{array}{c}\text { SpyCatcher-positive } \\
\text { Biotinylation-positive }\end{array}$} & \multicolumn{2}{c|}{$\begin{array}{c}\text { SpyCatcher-negative } \\
\text { Biotinylation-positive }\end{array}$} & \multicolumn{2}{c|}{$\begin{array}{c}\text { SpyCatcher-positive } \\
\text { Biotinylation-negative }\end{array}$} \\
\hline $\begin{array}{c}\text { Field of } \\
\text { view 1 }\end{array}$ & $\begin{array}{r}\text { Field of } \\
\text { view 2 }\end{array}$ & Field of & Field of 1 & view 2 & view 1 & vield of \\
\hline $\begin{array}{c}\text { Number of } \\
\text { pedestals }\end{array}$ & 146 & 163 & 89 & 137 & 188 & 168 \\
\hline $\begin{array}{c}\text { Number of } \\
\text { probe beads }\end{array}$ & 513 & 493 & 0 & 0 & 0 & 0 \\
\hline $\begin{array}{c}\text { Mean probe } \\
\text { beads per } \\
\text { pedestal }\end{array}$ & 3.5 & 3.0 & 0 & 0 & 0 & 0 \\
\hline
\end{tabular}


1214 Extended Data Table 3 | Sources of uncertainty in the photonic-force microscope and

1215 single-molecule experiment.

1216

\begin{tabular}{|c|c|c|}
\hline$b$ & $\begin{array}{l}\text { Probe position detector: precision } \\
\text { Over one extension-relaxation cycle (SD, } 1 \mathrm{~Hz}-1 \mathrm{MHz} \text { ) } \\
\text { Root-mean-square noise over } 5 \mathrm{~min}\end{array}$ & $\begin{array}{l}0.3 \mathrm{~nm} \\
2 \mathrm{~nm}\end{array}$ \\
\hline c & $\begin{array}{l}\text { Probe position detector: accuracy } \\
\text { Calibration error (SD computed from } 12 \text { independent probe beads) }\end{array}$ & $5 \%$ \\
\hline e & $\begin{array}{l}\text { Position of the stimulus trap relative to the position-sensing trap } \\
\text { Accuracy of location of origin (peak-to-peak) } \\
\text { Total accuracy (RMS., from (c) and (d) for a displacement of } 200 \mathrm{~nm} \text { ) } \\
\text { Drift }\end{array}$ & $\begin{array}{l} \pm 4 \mathrm{~nm} \\
10 \mathrm{~nm} \\
0.2 \mathrm{~nm} / \mathrm{min}\end{array}$ \\
\hline $\mathrm{h}$ & $\begin{array}{l}\text { Spring constant of the stimulus trap: precision } \\
\text { Root-mean-square noise over } 20 \mathrm{~s} \\
\text { Drift }\end{array}$ & $\begin{array}{l}0.27 \mu \mathrm{N} \cdot \mathrm{m}^{-1} \\
0 \mathrm{~N} \cdot \mathrm{m}^{-1} \cdot \mathrm{s}^{-1}\end{array}$ \\
\hline $\mathrm{i}$ & Spring constant of the stimulus trap: accuracy. & $5 \%$ \\
\hline j & $\begin{array}{l}\text { Force: precision (over } 5 \mathrm{~min} \text { for } 200 \mathrm{~nm} \text { displacement of the probe bead } \\
\text { from the stimulus trap and at maximum stimulus-laser power) } \\
\text { Total precision computed from (b), (f), and (g) }\end{array}$ & $0.7 \mathrm{pN}$ \\
\hline k & $\begin{array}{l}\text { Force: accuracy (for } 200 \mathrm{~nm} \text { displacement of the probe bead from the } \\
\text { stimulus trap and maximum stimulus laser power) } \\
\text { Total accuracy computed from (e) and (i) }\end{array}$ & $3.8 \mathrm{pN}$ \\
\hline
\end{tabular}




\section{Extended Data Notes}

\section{Extended Data Note 1 | Interpretation of State 1 as folded PCDH15}

1221 We verified that conformational State 1, that with the smallest contour length (Figure 2d,e),

1222 corresponds to fully folded PCDH15. First, we did not observe any reproducibly accessible states

1223 with contour lengths shorter than that of State 1. Although we sporadically observe individual

1224 force-extension relations to the left of State 1, these curves were not reproducible and likely

1225 reflected nonspecific interactions between the pedestal or probe bead surfaces and the protein.

1226 Second, fits of our polymer model to the data for each State 1 yielded contour lengths of $122746 \pm 7 \mathrm{~nm}$ and $47 \pm 7 \mathrm{~nm}$ for $\mathrm{Ca}^{2+}$ concentrations of respectively $3 \mathrm{mM}$ and $20 \mu \mathrm{M}$ (means \pm SEMs 1228 for respectively five and eight molecules). These values accord well with a contour length of $122950 \mathrm{~nm}$ expected for a chain of 11 cadherin domains, each $4.5 \mathrm{~nm}$ in length ${ }^{23}$.

1230 Extended Data Note 2 | Influence of possible molten-globule states on domain1231 unfolding rates.

1232 At tensions below $20 \mathrm{pN}$ and with $\mathrm{Ca}^{2+}$ concentrations of $20 \mu \mathrm{M}$ or zero, the rate of unfolding of 1233 cadherin domains diverges from an exponential relationship and systematically reaches higher 1234 values than expected (Figure 4). What causes this effect? It is possible that cadherin domains 1235 refold through a long-lived molten-globule state, as occurs for other proteins with 1236 immunoglobin-like folds ${ }^{60}$. During the waiting time between trials, an unfolded domain might 1237 refold completely, fold into the molten-globule state, or not refold at all. If folding occurred, the 1238 domain might unfold during the next extension from the completely folded state or from the 1239 molten-globule state. We cannot differentiate between these two possibilities, for they would 1240 result in indistinguishable contour-length changes. Molten globules are known to unfold readily 1241 at low forces ${ }^{60,61}$. Because such rapid unfolding events would increase our measured unfolding 1242 rates, they might explain the unexpected behavior observed at low tensions. 


\section{Extended Data Note 3 | An estimate for the enthalpic stiffness of full-length, dimeric tip}

\section{4 links.}

1245 A full-length tip link consists of a dimer of PCDH15, which forms the lower one-third of the 1246 filament, and a dimer of $\mathrm{CDH} 23$ that makes up the upper two-thirds of its length. For monomeric 1247 PCDH15 we found an enthalpic stiffness of $9 \mathrm{mN} \mathrm{m}^{-1}$. A PCDH15 dimer, made up of two 1248 monomers in parallel, then has a stiffness of roughly $18 \mathrm{mN} \mathrm{m}^{-1}$. If $\mathrm{CDH} 23$ has a similar enthalpic 1249 stiffness per length, then the full-length dimeric tip link has a stiffness of one-third of that of 1250 dimeric PCDH15, resulting in an estimate of $6 \mathrm{mN} \mathrm{m}^{-1}$. 